\title{
COMPLEX VECTOR FIELDS HAVING ORBITS WITH BOUNDED GEOMETRY
}

\author{
BRUNO C. A. SCÁRduA \\ (Received January 11, 1999, revised February 12, 2002)
}

\begin{abstract}
Germs of holomorphic vector fields at the origin $0 \in \boldsymbol{C}^{2}$ and polynomial vector fields on $\boldsymbol{C}^{2}$ are studied. Our aim is to classify these vector fields whose orbits have bounded geometry in a certain sense. Namely, we consider the following situations: (i) the volume of orbits is an integrable function, (ii) the orbits have sub-exponential growth, (iii) the total curvature of orbits is finite. In each case we classify these vector fields under some generic hypothesis on singularities. Applications to questions, concerning polynomial vector fields having closed orbits and complete polynomial vector fields, are given. We also give some applications to the classical theory of compact foliations.
\end{abstract}

1. Introduction. Let $\bar{M}$ be a manifold with a foliation $\mathcal{F}$. We equip $\bar{M}$ with a Riemannian metric $d s^{2}$, and for each leaf $L \subset \bar{M}$ consider the isometric immersion given by the inclusion. It has been shown by several authors that for nonsingular real foliations their global behavior is often related to the geometric properties of isometrically embedded leaves ([12], [13], [28], [29]). In many cases, this global behavior depends on finiteness conditions on the geometry of leaves. In this paper we are concerned with similar problems for complex (possibly) singular foliations, defined for instance by a (singular) holomorphic vector field on a manifold ([20]).

The following theorems are main results proved in this paper.

THEOREM 1. Let $X$ be a germ of holomorphic vector field in an open neighborhood $U$ of the origin $0 \in \boldsymbol{C}^{2}$. Assume that the Euclidean volume function of $X$ is integrable and that the total volume of the orbits accumulating to the origin is finite. Then $\mathcal{F}$ has a holomorphic first integral.

For the definition of the volume function of a vector field $X$, see Section 3 .

THEOREM 2. Let $X$ be a polynomial vector field in $\boldsymbol{C}^{2}$. Assume that the Fubini-Study volume function of $X$ is integrable in $C^{2}$ and that the singularities of the corresponding projective foliation $\mathcal{F}(X)$ of $\boldsymbol{C} P(2)$ in $L_{\infty}$ are of rational type. Then $\mathcal{F}(X)$ admits a rational first integral.

2000 Mathematics Subject Classification. Primary 32L30; Secondary 58F18.

Key words and phrases. Singular holomorphic foliation, bounded geometry, holonomy group.

The author is grateful to Professor César Camacho for encouraging conversation. The author is a fellow of CNPq-Brasil. 
For the notion of rational type singularity, see Section 2. Theorems 1 and 2 are related to the main result of [1], which is in a local nature.

Given a polynomial vector field $X$ in $C^{2}$ there is an induced foliation $\mathcal{F}=\mathcal{F}(X)$ on the complex projective plane $\boldsymbol{C P}(2)=\boldsymbol{C}^{2} \cup L_{\infty}$ ([20]). Denote by $\operatorname{sing} \mathcal{F} \subset \boldsymbol{C P}(2)$ the singular set of $\mathcal{F}$. Note that the line at the infinity $L_{\infty} \cong C P(1)$ must have some singularity of $\mathcal{F}$ ([20]).

THEOREM 3. Let $\mathcal{F}$ be a foliation by curves on $C P(2)$ whose singularities in $L_{\infty}$ are of rational type. If the leaves of $\left.\mathcal{F}\right|_{C^{2}}$ are closed in $C^{2} \backslash \operatorname{sing} \mathcal{F}$, then $\mathcal{F}$ admits a rational first integral.

The above result is analogous to the theorem of Mattei-Moussu for germs of foliations ([22]). For the next theorem we need the notion of growth of leaves introduced in [28].

THEOREM 4. Let $\mathcal{F}$ be a foliation on $\boldsymbol{C P}(2)$, whose singularities on $\boldsymbol{C P}(2)$ are hyperbolic and whose leaves have sub-exponential growth, for some $C^{\infty}$ metric $g$ on $C P(2)$ which is Hermitian along the leaves. Then $\mathcal{F}$ is linear of the form $\dot{x}=x, \dot{y}=\lambda y, \lambda \in \boldsymbol{C} \backslash \boldsymbol{R}$, with respect to some affine coordinates $(x, y) \in \boldsymbol{C}^{2} \subset \boldsymbol{C P}(2)$. In particular, the limit set of $\mathcal{F}$ is a union of singularities and algebraic leaves.

Theorem 4 gives rise to, from the standpoint of Plante ([28]), a variant of PoincaréBendixson type theorem for polynomial vector fields.

THEOREM 5. Let $X$ be a polynomial vector field in $C^{2}$, whose corresponding projective foliation $\mathcal{F}(X)$ has only hyperbolic singularities in $C P(2)$. Assume that the orbits of $X$ have finite total curvature for the isometric immersion given by the inclusion. Then $X$ is linear $X=\lambda x(\partial / \partial x)+\mu y(\partial / \partial y), \lambda / \mu \in \boldsymbol{C} \backslash \boldsymbol{R}$, in some affine chart.

Theorem 5 concerns a question related to minimal immersions in Euclidean spaces (see Osserman [27]).

In the last section of the paper we consider compact foliations (that is, foliations on compact manifolds by compact leaves), and study the basic questions in a classical framework.

It should be remarked that our global approach is based on the study of the holonomy groups associated to some algebraic invariant curve on $C P(2)$, to which we extend the foliation induced by any polynomial vector field on $C^{2}$. This algebraic curve may be the line at the infinity $L_{\infty}=\boldsymbol{C P}(2) \backslash \boldsymbol{C}^{2}$, or some other algebraic leaf which exists depending on the context. These holonomy groups are proved to be finite, abelian linearizable or solvable affine groups depending on the situation we deal with, as a consequence of our hypothesis of bounded geometry. Then the classification of these foliations is obtained by applying the main results in [5], [8], [31] and [33].

Although the results stated above have geometric interpretations, our approach stems from complex dynamics and geometric theory of foliations. It is interesting to exploit such 
machinery in further investigation of geometric questions such as (i) the existence of exceptional minimal sets for holomorphic foliations in $\boldsymbol{C P}$ (2) ([6]), (ii) polynomial vector fields with simply connected orbits in $\boldsymbol{C}^{n}$ ([11]), and others.

I would like to thank the referee of the original manuscript for the careful reading and suggestions, which helped improve the final form of this paper. The first part of this work was conceived during my visit to the IMCA-Peru. I wish to thank my hosts for their hospitality.

2. Preliminaries. In this section we introduce basic notation and relevant facts from complex dynamics for later use. We refer to [22], [31] and [33] for further specific information.

2.1. Groups with finite orbits ([22]). Let $\operatorname{Diff}(\boldsymbol{C}, 0)$ denote the group of germs of holomorphic diffeomorphisms at the origin $0 \in \boldsymbol{C}$. We denote by $\boldsymbol{C}^{*}=\boldsymbol{C} \backslash\{0\}$. For each $k \in N$ we define the group

$$
\boldsymbol{H}_{k}=\left\{\varphi \in \operatorname{Diff}(\boldsymbol{C}, 0) ; \varphi(z)^{k}=\frac{\mu_{\varphi} z^{k}}{1+a_{\varphi} z^{k}}, \mu_{\varphi} \in \boldsymbol{C}^{*}, a_{\varphi} \in \boldsymbol{C}\right\} .
$$

Then $\boldsymbol{H}_{k}$ is a solvable group and, up to formal conjugacy, any solvable nonabelian subgroup $G$ of $\operatorname{Diff}(\boldsymbol{C}, 0)$ is of this type ([21]) (a result by Cerveau-Moussu). If this conjugacy is analytic, then the group is called analytically normalizable (see [9], [33] for the general notion of analytic normalizable group). This analytic normalization can be carried out except for some special cases where the group of commutator $[G, G]$ is cyclic ([21]). Such non-analytically normalizable groups are called exceptional. The following is a generalization of a result in [22].

Lemma 1. Let $G \subset \operatorname{Diff}(\boldsymbol{C}, 0)$ be a finitely generated subgroup such that for almost every point $p$ (with respect to the Euclidean Lebesgue measure) in a neighborhood $\Omega$ of $0 \in \boldsymbol{C}$ the pseudo-orbit of $p$ is finite. Then $G$ is finite and analytically conjugate to a cyclic group of rational rotations.

Proof. First we observe that by Nakai density theorem ([26]) the group $G$ must be solvable. On the other hand, if $G$ is nonabelian, then we may take an element $g \in G$ of the form $g(z)=z+a_{k+1} z^{k+1}+$ h.o.t., $a_{k+1} \neq 0$. According to [3] the pseudo-orbits of this element are not finite. Thus $G$ must be abelian and cannot contain elements of the form $g(z)=z+a_{k+1} z^{k+1}+$ h.o.t., $g \neq$ Id.

\section{Claim 1. Any element $g \in G$ has finite order.}

Indeed, assume that there exists $g \in G$ with $g^{n} \neq$ Id for any $n \in \boldsymbol{Z}-\{0\}$. We may assume that $g(z)=\lambda \cdot z+$ h.o.t., $\lambda^{n} \neq 1$ for any $n \in \boldsymbol{Z}-\{0\}$. If $|\lambda| \neq 1$, then by Poincaré linearization theorem we may linearize $g$ or $g^{-1}$ as an attractor so that none of its pseudoorbits is finite. Thus $|\lambda|=1$ and we have $\lambda=\exp (2 \pi \sqrt{-1} \alpha)$ for some $\alpha \in \boldsymbol{R} \backslash \boldsymbol{Q}$. This implies that the pseudo-orbits of $g$ are not finite, which is a contradiction and proves the claim.

Since $G$ is abelian and finitely generated, the claim implies that $G$ itself is finite. According to [22], $G$ must be a group of rational rotations up to analytic conjugacy. 
2.2. Singularities in dimension two ([22], [20]). Given a foliation $\mathcal{F}$ of dimension one on a complex surface $\bar{M}$ with singular set sing $\mathcal{F}$, the reduction theorem of Seidenberg ([35]) (see [10] for a version in dimension three) asserts the existence of a proper holomorphic map $\pi: \tilde{M} \rightarrow \bar{M}$ which is a finite composition of quadratic blowing-up's at the singular points of $\mathcal{F}$ in $\bar{M}$ such that the pull-back foliation $\tilde{\mathcal{F}}:=\pi^{*} \mathcal{F}$ of $\mathcal{F}$ by $\pi$ satisfies:

(a) $\operatorname{sing} \tilde{\mathcal{F}} \subset \pi^{-1}(\operatorname{sing} \mathcal{F})$, and

(b) any singularity $\tilde{p} \in \operatorname{sing} \tilde{\mathcal{F}}$ belongs to one of the following categories (these are called irreducible singularities):

(i) $x d y-\lambda y d x+$ h.o.t. $=0$ and $\lambda$ is not a positive rational number, i.e., $\lambda \notin \boldsymbol{Q}_{+}$ (simple singularity),

(ii) $y^{p+1} d x-\left[x\left(1+\lambda y^{p}\right)+\right.$ h.o.t. $] d y=0, p \geq 1$. This case is called a saddle-node. We call $\tilde{\mathcal{F}}$ the desingularization or reduction of singularities of $\mathcal{F}$.

Definition 1. A singularity of type (i) is called resonant if $\lambda$ is negative rational number, i.e., $\lambda \in \boldsymbol{Q}_{-}$([23]). An isolated singularity of $\mathcal{F}$ is called a generalized curve if the desingularization $\tilde{\mathcal{F}}$ of $\mathcal{F}$ admits no saddle-node singularities.

The exceptional divisor $D=\pi^{-1}(\operatorname{sing} \mathcal{F}) \subset \tilde{M}$ of the resolution $\pi$ can be written as $D=\bigcup_{j=1}^{m} D_{j}$, where each $D_{j}$ is diffeomorphic to an embedded projective line $\boldsymbol{C P}(1)$ introduced as a divisor of the successive blowing-up's. $D_{j}$ are called components of the divisor $D$. A singularity $q \in \operatorname{sing} \mathcal{F}$ is nondicritical if $\pi^{-1}(q)$ is invariant by $\tilde{\mathcal{F}}$. Any two $D_{i}$ and $D_{j}, i \neq j$, intersect (transversely) at at most one point, which is called a corner. There are no triple intersection points.

Given any analytic curve $\Gamma \subset \bar{M}$ we denote by $\tilde{\Gamma}:=\overline{\pi^{-1}(\Gamma \backslash \operatorname{sing} \mathcal{F})} \subset \tilde{M}$ the strict transform of $\Gamma$. Let now $p$ be a singularity of a germ of foliation admitting a germ of smooth separatrix $S$. A linear chain at $p$ (with respect to $S$ ) is a sequence of blowing-up's constructed in the following way: Let $\pi_{1}$ be a blowing-up at $p$ and $P_{1}=\pi_{1}^{-1}(p)$. If $p_{1}=\pi^{*}(S) \cap P_{1}$ is reduced (where $\pi^{*}(S)$ denotes the strict transform of $S$ under $\pi_{1}$ ), then the linear chain at $p_{1}$ is $\pi_{1}$. If $p_{1}$ is not reduced, then we consider another blowing-up $\pi_{2}$ at $p_{1}$, and if necessary, successive blowing-up's at the corners, until all of them are reduced. The linear chain at $p$ consists of the composition $\pi_{r} \circ \cdots \circ \pi_{1}$ of all these blowing-up's (see [7], [25] for further information).

A dicritical singularity $q \in \operatorname{sing} \mathcal{F} \cap S$ (where $S$ is as above) will be called ordinary dicritical (with respect to $S$ ) if only one component of $\pi^{-1}(q)$ is noninvariant and appears in the first linear chain of $q$ (with respect to $S$ ).

We will mainly consider nondicritical singularities. However we do admit dicritical singularities of the following type:

Definition 2 (rational singularity [25]). Let $\mathcal{F}$ be a foliation on $\boldsymbol{C P}(2)$. Given a smooth algebraic curve $\Lambda \subset \boldsymbol{C P}(2)$ we say that the singularities of $\mathcal{F}$ in $\Lambda$ are of rational type if: 
(i) either $\Lambda$ is not invariant by $\mathcal{F}$, or the complement of the resolution divisor $D$ of $\operatorname{sing} \mathcal{F} \cap \Lambda$ is pseudo-convex (see [9] for sufficient conditions for pseudo-convexity).

(ii) the singularities $\operatorname{sing} \mathcal{F} \cap \Lambda$ are generalized curves (see Definition 1 above) and one of the following conditions is satisfied:

(ii.1) $\operatorname{sing} \mathcal{F} \cap \Lambda$ has a nondicritical singularity.

(ii.2) $\operatorname{sing} \mathcal{F} \cap \Lambda=\{q\}$ and $q$ is ordinary dicritical (with respect to $\Lambda$ ).

(ii.3) $\operatorname{sing} \mathcal{F} \cap \Lambda$ contains at least $n+1$ ordinary dicritical singularities, where $n \geq 0$ is the self-intersection number of $\Lambda \subset \boldsymbol{C P}(2)$.

Conditions (ii.2) and (ii.3) are assumed when all singularities in $\Lambda$ are dicritical. If $\Lambda=L_{\infty}$, the conditions above exclude the case where $\operatorname{sing} \mathcal{F} \cap \Lambda$ consists of more than two dicritical singularities but only one of them is ordinary dicritical. One of the main results in [25] is the following

THEOREM 6. Let $\mathcal{F}$ be a singular holomorphic foliation by curves on a complex surface $M, S \subset M$ an invariant analytic (irreducible) compact curve. Then the foliation $\mathcal{F}$ has some separatrix transverse to $S$ provided that one of the following conditions is satisfied:

(i) Some singularity $p \in \operatorname{sing} \mathcal{F} \cap S$ is a non-dicritical generalized curve.

(ii) $S$ is smooth with negative self-intersection number and each $p \in \operatorname{sing} \mathcal{F} \cap S$ is a generalized curve.

(iii) $S$ is smooth with self-intersection number $n \geq 0$, each $p \in \operatorname{sing} \mathcal{F} \cap S$ is a generalized curve, and at least $n+1$ of them are ordinary dicritical.

In particular, if $\mathcal{F}$ admits a meromorphic first integral $\xi: M \backslash S \rightarrow \overline{\boldsymbol{C}}$, then $\xi$ extends to a meromorphic first integral on $M$ provided that one of the conditions above is satisfied.

We apply this result in the proof of Theorem 2 as well as Proposition 4 to find some separatrix transverse to $L_{\infty}$ in $\boldsymbol{C P}(2)$ or to extend some meromorphic first integral to a rational function.

Examples of ordinary dicritical singularities include singularities admitting a local meromorphic first integral of the form $x^{n} / y^{m}$. Note that singularities admitting holomorphic first integrals are always nondicritical ([22]).

2.3. Adjunction of holonomy groups ([33]). We consider the following situation motivated by the above reduction theorem: $\tilde{\mathcal{F}}$ is a foliation on a compact complex surface $\tilde{M}$, and $D \subset \tilde{M}$ is a compact (codimension one) invariant divisor with normal crossing and no triple points. We write $D=\bigcup_{j=1}^{m} D_{j}$, where each $D_{j}$ is an irreducible smooth component, and fix local transverse sections $\mathcal{S}_{j}$ such that $\mathcal{S}_{j} \cap D_{j}=p_{j} \notin \operatorname{sing} \tilde{\mathcal{F}}$, and $\left(\mathcal{S}_{j}, p_{j}\right) \cong(\boldsymbol{C}, 0)$. Denote by $G_{j}$ the holonomy group $\operatorname{Hol}\left(\tilde{\mathcal{F}}, D_{j}, \mathcal{S}_{j}\right.$ ) of $D_{j}$ (we refer to [22] for the definition and properties of the holonomy group). Denote by $\tilde{L}_{z}$ the leaf of $\tilde{\mathcal{F}}$ that contains the point 
$z \in \tilde{M}$. The virtual holonomy group $\hat{G}_{j}$ of $\tilde{\mathcal{F}}$ relative to the component $D_{j}$ at the section $\mathcal{S}_{j}$ is defined to be ([5])

$$
\hat{G_{j}}=\hat{\operatorname{Hol}}\left(\tilde{\mathcal{F}}, D_{j}, \mathcal{S}_{j}\right)=\left\{f \in \operatorname{Diff}\left(\mathcal{S}_{j}, p_{j}\right) \mid \tilde{L}_{z}=\tilde{L}_{f(z)} \text {, for any } z \in\left(\mathcal{S}_{j}, p_{j}\right)\right\} .
$$

Clearly, this virtual holonomy group $\hat{G}_{j}$ contains the holonomy group $G_{j}$. Now we fix a corner $q=D_{i} \cap D_{j}$, and make the following assumption:

Assumption 1. The virtual holonomy group $\hat{G}_{i}$ is solvable and the group $\hat{G}_{j}$ is finite.

Thus, in the nonabelian case we have $\hat{G}_{i} \subset \boldsymbol{H}_{k_{i}}$ for some formal embedding. We now define the $G_{i}$ to $G_{j}$, which is, roughly speaking, a consequence of passing one holonomy group to the other adjacent transverse section, by using the Dulac correspondence, as follows (see also [9], [11] and [33] for more details and the original construction): Choose a neighborhood $U$ of $q$, where $\tilde{\mathcal{F}}$ can be written in the normal form. In $U$, we take small transverse sections $\Sigma_{i}, \Sigma_{j} \subset U$ to $\tilde{\mathcal{F}}$ such that $\Sigma_{i} \cap D_{i}=q_{i}$ and $\Sigma_{j} \cap D_{j}=q_{j}$. Denote by $\mathcal{F}\left(\Sigma_{i}\right)$ the collection of subsets $E \subset \Sigma_{i}$ such that $E$ is contained in some leaf of $\left.\tilde{\mathcal{F}}\right|_{U}$. Define $\mathcal{F}\left(\Sigma_{j}\right)$ in a similar way. The Dulac correspondence is a multivalued correspondence $\mathcal{D}_{q}: \mathcal{F}\left(\Sigma_{i}\right) \rightarrow \mathcal{F}\left(\Sigma_{j}\right)$, which is obtained by tracing the local leaves of $\left.\tilde{\mathcal{F}}\right|_{U}$. Given any $x \in \Sigma_{i}$ the set of intersections of the local leaf of $\left.\tilde{\mathcal{F}}\right|_{U}$ that contains $x$, with the transverse section $\Sigma_{i}$, is denoted by $\tilde{L}_{x} \cap \Sigma_{i} \in \mathcal{F}\left(\Sigma_{i}\right)$. The correspondence $\mathcal{D}_{q}$ associates to any point $z \in \tilde{L}_{x} \cap \Sigma_{i}$, a subset $\mathcal{D}_{q}(z) \subset \tilde{L}_{x} \cap \Sigma_{j} \in \mathcal{F}\left(\Sigma_{j}\right)$, that is defined by the local normal form of $\tilde{\mathcal{F}}$ in $U$.

Given an element $h \in \operatorname{Hol}\left(\tilde{\mathcal{F}}, D_{i}, \Sigma_{i}\right)$, we associate $h$ with a collection of elements $\left\{h^{\mathcal{D}}\right\} \subset \operatorname{Diff}\left(\Sigma_{j}, q_{j}\right)$, each of which satisfies the following relation

$$
h^{\mathcal{D}} \circ \mathcal{D}_{q}=\mathcal{D}_{q} \circ h,
$$

called the adjunction equation. Remark that the adjunction equation is not exactly an equation but an equality of sets or correspondences. More precisely, given any element $h \in$ $\operatorname{Hol}\left(\tilde{\mathcal{F}}, D_{i}, \Sigma_{i}\right)$, each diffeomorphism $h^{\mathcal{D}} \in \operatorname{Diff}\left(\Sigma_{j}, q_{j}\right)$ must satisfy, for every $x \in \Sigma_{i}$, the equality of sets $h^{\mathcal{D}}\left(\mathcal{D}_{q}(x)\right)=\mathcal{D}_{q}(h(x))$, where $\mathcal{D}_{q}(x) \subset \tilde{L}_{x} \cap \Sigma_{i}$ and $\mathcal{D}_{q}(h(x)) \subset \tilde{L}_{x} \cap \Sigma_{j}$ are subsets as above. This adjunction is adequately defined for the special case of singularities $\{q\}=D_{i} \cap D_{j}$ we consider below.

Up to a conjugacy induced by a simple path in $D_{i} \backslash \operatorname{sing} \tilde{\mathcal{F}}$ joining $p_{i}$ to $q_{i}$, we can assume that $G_{i}=\operatorname{Hol}\left(\tilde{\mathcal{F}}, D_{i}, \Sigma_{i}\right)$ and $G_{j}=\operatorname{Hol}\left(\tilde{\mathcal{F}}, D_{j}, \Sigma_{j}\right)$. Therefore, the adjunction equation induces a subgroup $G_{j} *\left(\mathcal{D}_{q_{*}} G_{i}\right) \subset \operatorname{Diff}\left(\mathcal{S}_{j}, p_{j}\right)$ which is generated by $G_{j}$ and all elements $h^{\mathcal{D}_{q}}, h \in G_{i}$. This subgroup is unique up to above conjugacies. We observe that the subgroup $G_{j} *\left(\mathcal{D}_{q_{*}} G_{i}\right)$ is not necessarily finitely generated.

For our purpose we may assume the following:

AsSUMPTION 2. The corner $q$ is a singularity with a holomorphic first integral.

Therefore there are local holomorphic coordinates $(x, y) \in U$ such that $D_{i} \cap U=\{x=$ $0\}, D_{j} \cap U=\{y=0\}$, and such that $\left.\tilde{\mathcal{F}}\right|_{U}$ is given in the normal form as $n x d y+m y d x=0$ 
and $q: x=y=0$, where $n / m \in Q_{+}$and $\langle n, m\rangle=1$. We fix the local transverse sections as $\Sigma_{j}=\{x=1\}$ and $\Sigma_{i}=\{y=1\}$, such that $\Sigma_{i} \cap D_{i}=q_{i} \neq q$ and $\Sigma_{j} \cap D_{j}=q_{j} \neq$ $q$. Denote by $h_{o} \in \operatorname{Hol}\left(\tilde{\mathcal{F}}, D_{i}, \Sigma_{i}\right)$ the element corresponding to the corner $q$. Then we have $h_{o}(x)=\exp (-2 \sqrt{-1} \pi n / m) x$. The local leaves are given by $x^{m} y^{n}=$ cte. The Dulac correspondence is therefore given by (all branches are considered)

$$
\mathcal{D}_{q}: \mathcal{F}\left(\Sigma_{i}\right) \rightarrow \mathcal{F}\left(\Sigma_{j}\right), \quad \mathcal{D}_{q}(x)=\left\{x^{m / n}\right\} .
$$

Case 1. $G_{i}$ is abelian: Take any element $h \in G_{i}$. Since $G_{i}$ is abelian, we have $h(x)=$ $\mu x \tilde{h}\left(x^{m}\right)$ for some $\tilde{h} \in \mathcal{O}_{1}, \tilde{h}(0)=1$. We take $\mu_{1}=\mu^{m / n}$ and $h_{1}=\tilde{h}^{m / n}$ to be one of the $n$-roots of $\mu^{m}$ and $\tilde{h}^{m}$, respectively. Then we define $h^{\mathcal{D}_{q}}:\left(\Sigma_{j}, q_{j}\right) \rightarrow\left(\Sigma_{j}, q_{j}\right)$ by $h^{\mathcal{D}_{q}}(y)=\mu_{1} y h_{1}\left(y^{n}\right)$. We consider the collection $\left\{h^{\mathcal{D}}\right\}$ of all these elements.

Case 2. $\quad G_{i} \subset \boldsymbol{H}_{k_{i}}$ is nonabelian, analytically normalizable and $n k_{i} / m=k_{j} \in N$ : In this case we have an analytic embedding $G_{i} \subset \boldsymbol{H}_{k_{i}}$. Take an element

$$
h(x)=\lambda x /\left(1+a x^{k_{i}}\right)^{1 / k_{i}} \in G_{i},
$$

and consider the collection $\left\{h^{\mathcal{D}}\right\}$ of all branches of

$$
h^{\mathcal{D}_{q}}: y \mapsto \lambda^{m / n} y /\left(1+a y^{n k_{i} / m}\right)^{m / n k_{i}} .
$$

Case 3. $G_{i}$ is solvable, nonabelian and not analytically normalizable: In this case it follows from [21] that the group of commutators $\left[G_{i}, G_{i}\right]$ is cyclic, that is, $\left[G_{i}, G_{i}\right]=\left\langle h_{1}\right\rangle$ for some $h_{1} \in G_{i}$ and $G_{i}$ is generated by some power or root of local holonomy map $h_{o}$ (associated to the singularity $q=D_{i} \cap D_{j}$ ) and some power $h_{1}^{l}, l \in Z$. Notice that if $n=m=1$, then the local leaves are given by $x y=$ cte and we have $\mathcal{D}_{q}(x)=\left\{x^{n / m}\right\}=\{x\}$. Thus, in this case, $\mathcal{D}_{q}$ is a map $\mathcal{D}_{q}: \Sigma_{i} \rightarrow \Sigma_{j}$. Given any $h \in G_{i}$, we may therefore define $h^{\mathcal{D}_{q}} \in \hat{G}_{j}$ as $h^{\mathcal{D}_{q}}(y)=h(y)$ in the coordinates defined above. Hence we may assume $n \neq m$.

First, consider the case where the virtual holonomy group $\hat{G}_{j}$ is abelian. Then all elements of $\hat{G}_{j}$ commute with the local holonomy $g_{o}$ around $q$, associated to the separatrix contained in $D_{j}$. Therefore, using the same construction as in Case 1 above, we may consider the adjunction of $\hat{G}_{j}$ to the holonomy group $G_{i}$, as a subgroup of the virtual holonomy group $\hat{G}_{i}$. If $\hat{G}_{j}$ contains some element $g$ of infinite order, then we have two possibilities to consider:

(3.a) $g$ has nonperiodic linear part. In this case, $g$ induces an element $h$ in the adjunction holonomy and therefore in the virtual holonomy $h \in \hat{G}_{i}$, which also has nonperiodic linear part. This implies that $\hat{G}_{i}$ (which is solvable by hypothesis) is analytically normalizable [21]. Therefore we may exclude this case.

(3.b) Every element $g$ in $\hat{G}_{j}$ has periodic linear part. In this case, we may find some nontrivial element $g \in \hat{G}_{j}$, which is tangent to the identity $g(y)=y+a y^{l+1}+$ h.o.t., $a \neq 0$. Then, $g$ induces an element $h$ in the virtual holonomy $\hat{G}_{i}$, which has infinite order and some power tangent to the identity. Moreover, since $\hat{G}_{j}$ is analytically normalizable, it follows that $g$ and $h$ are analytically normalizable. This implies that the powers of $h$ are analytically normalizable and therefore, since the group of flat elements in $\hat{G}_{i}$ is cyclic, $\hat{G}_{i}$ is analytically 
normalizable. Hence we exclude this case, and conclude that all elements in $\hat{G}_{j}$ have finite order. It follows that $\hat{G}_{j}$ is a group whose elements are rational rotations, and that each finitely generated subgroup is in fact a finite analytically linearizable group. In particular, $G_{j}$ is a finite linearizable group.

Now we consider the case that $\hat{G}_{j}$ is solvable nonabelian, and analytically normalizable. In this case, once again we may use the same procedure as in Case 2 above to induce nontrivial analytically normalizable flat elements in the virtual holonomy $\hat{G}_{i}$, and conclude that this is in fact analytically normalizable. Thus we exclude this case, too.

Summing up, we obtain the following:

CONCLUSION. Under our assumptions above, if $G_{i}$ is exceptional (that is, solvable nonabelian and not analytically normalizable), then $\hat{G}_{j}$ is either a group of rational rotations and hence with a finite number of finitely generated subgroups, or an exceptional group.

In this last (exceptional) case, it follows from [21] that $\hat{G}_{j}$ is generated by some root of the local holonomy $g_{o}$ associated to $q$ (we may have $g_{o}=\mathrm{Id}$ ), and some flat element $g_{1}$. Moreover each flat element in $\hat{G}_{j}$ is some power of $g_{1}$. Then ideas in [26] and [9] conclude that certain power of $h_{1}$ corresponds to some power of $g_{1}$ by means of the Dulac correspondence. Indeed, it is possible to use the Dulac correspondence given by $\mathcal{D}_{q}(x)=$ $\left\{x^{m / n}\right\}$, in order to consider the sets of "pseudo-orbits"

$$
\left\{g_{1}^{k_{r}} \circ \mathcal{D}_{q} \circ h_{1}^{l_{r}} \circ \mathcal{D}_{q}^{-1} \circ \cdots \circ g_{1}^{k_{2}} \circ \mathcal{D}_{q} \circ h_{1}^{l_{2}} \circ \mathcal{D}_{q}^{-1} \circ g_{1}^{k_{1}} \circ \mathcal{D}_{q} \circ h_{1}^{l_{1}}(x)\right\} \subset \Sigma_{i},
$$

where $x \in \Sigma_{i}, l_{t}$ and $k_{t}$ are integers, and $\mathcal{D}_{q}^{-1}$ is the correspondence $\mathcal{F}\left(\Sigma_{j}\right) \rightarrow \mathcal{F}\left(\Sigma_{i}\right), y \mapsto$ $\left\{y^{n / m}\right\}$. These sets are contained in the same leaf of $\mathcal{F}$ for each fixed $x$, and as in [26], if the powers $g_{1}^{l}$ and $h_{1}^{k}$ are never related by the conjugacy equation (adjunction equation), then we have accumulations for the leaves of $\mathcal{F}$, outside the origin in $\Sigma_{i}$. On the other hand, in the case we are interested in, we have discrete intersections of the leaves with the transverse sections, outside the origin, so that some power $h_{1}^{l}$ passes to the virtual holonomy $\hat{G}_{j}$, as some power $g_{1}^{k}$.

3. Vector fields with integrable volume function. In this section we prove Theorems 1, 2 and 3. We consider the following situation: Let $X$ be a meromorphic vector field in a complex manifold $\bar{M}$, with polar set $(X)_{\infty}$ of codimension $\geq 1$, and discrete singular set $\operatorname{sing} X$. Thus $X$ induces a (singular) holomorphic foliation $\mathcal{F}(X)$ of dimension one on $\bar{M}$, whose leaves are integral curves of $X$ in $\bar{M} \backslash \operatorname{sing} X$ ([20]). The singular set $\operatorname{sing} \mathcal{F}(X)$ of $\mathcal{F}(X)$ is an analytic subset of codimension $\geq 2$. For each point $x \in \bar{M}$ we denote by $L_{x} \subset \bar{M} \backslash \operatorname{sing} \mathcal{F}(X)$ the corresponding leaf, and by $\mathcal{O}_{x} \subset L_{x}$ the associated orbit of $X$ in $\bar{M} \backslash(X)_{\infty}$. Let $\bar{M}$ be equipped with a Riemannian metric $d s^{2}$, which is Hermitian along the leaves of $\mathcal{F}(X)$. The volume function of $X$ (with respect to the metric $d s^{2}$ ) is therefore defined by

$$
\operatorname{Vol}_{X}: \bar{M} \rightarrow[0,+\infty], \quad \operatorname{Vol}_{X}(x)=\int_{L_{x}} i_{x}^{*}\left(d s^{2}\right),
$$


where $i_{x}: L_{x} \rightarrow \bar{M}$ is the inclusion map. We assume that $\operatorname{Vol}_{X}$ is an integrable function or that some open leaf of $\mathcal{F}(X)$ has finite volume. For technical reasons we avoid some exceptional singularities and work only with rational type singularities.

Let us now recall some basic facts on the geometry of the complex projective plane $\boldsymbol{C P}(2)$. The Fubini-Study metric of $\boldsymbol{C P}(2)$ is defined as

$$
d s^{2}=4\left(|d x|^{2}+|d y|^{2}+|x d y-y d x|^{2}\right) /\left(1+|x|^{2}+|y|^{2}\right)^{2},
$$

and the associated quadratic 2-form is written as

$$
\Omega=-4 \sqrt{-1} \partial \bar{\partial} \log \left(1+|x|^{2}+|y|^{2}\right) .
$$

Given a foliation $\mathcal{F}$ by curves in $\boldsymbol{C P}(2)$ and a leaf $L \subset \boldsymbol{C P}(2) \backslash \operatorname{sing} \mathcal{F}$, the area of a regular domain $D \subset L$ is given by

$$
A(D)=\left.\frac{1}{2 \pi} \int_{D} \Omega\right|_{L}
$$

For any local parametrization $\varphi(z)=(x(z), y(z)): \boldsymbol{D} \subset \boldsymbol{C} \rightarrow L$ of $L$, by setting $\psi(z)=$ $1+|x(z)|^{2}+|y(z)|^{2}$, we find that

$$
4 \bar{\partial} \partial \log \psi=\Delta \log \psi=\left[\psi \Delta \psi-|\operatorname{grad} \psi|^{2}\right] / \psi^{2} .
$$

Before proving Theorems 1 and 2, some examples illustrating these results are in order.

EXAMPLE 1. Let $R: \boldsymbol{C}^{2} \subset \boldsymbol{C P}(2) \rightarrow \boldsymbol{C P}(1)$ be a (nonconstant) rational function. The levels of $R$ define a foliation $\mathcal{F}$ by algebraic curves in $C P(2)$. Given any affine space $C^{2} \subset C P(2)$, there exists a polynomial vector field whose orbits are punctured leaves of $\mathcal{F}$ in $C^{2}$. The rational vector field $X=\left(-R_{y}, R_{x}\right)$ defines $\mathcal{F}$ outside its polar set. For any leaf $L$, the induced metric is written as

$$
\begin{aligned}
\mu^{2} & =4\left(\left|x^{\prime}(z)\right|^{2}+\left|y^{\prime}(z)\right|^{2}+\left|x(z) y^{\prime}(z)-x^{\prime}(z) y(z)\right|^{2}\right) /\left(1+|x(z)|^{2}+|y(z)|^{2}\right)^{2}|d z|^{2} \\
& =4\left(\left|x^{\prime}(z)\right|^{2}+\left|y^{\prime}(z)\right|^{2}\right) /\left(1+|x(z)|^{2}+|y(z)|^{2}\right)^{2}|d z|^{2} .
\end{aligned}
$$

Since the leaves are algebraic subvarieties of $\boldsymbol{C P}(2)$, the leaf space $(\boldsymbol{C P}(2) \backslash \operatorname{sing} \mathcal{F}) / \mathcal{F}$ is a nonsingular orbifold (a compact analytic space) and therefore (as in [1]) by Fubini's theorem the integral of the volumes of leaves (fibers of $R$ ) is integrable over $\boldsymbol{C P}(2)$.

EXAMPLE 2. Let us take a linear vector field $X(x, y)=x(\partial / \partial x)+\lambda y(\partial / \partial y), \lambda \in$ $\boldsymbol{C}^{*}$, where $(x, y)$ are affine coordinates in $\boldsymbol{C}^{2}$. The orbit $\mathcal{O}_{(x, y)}$ through a point $(x, y)$ is parametrized by $\varphi_{(x, y)}(z)=\left(x e^{z}, y e^{\lambda z}\right), z \in D(\lambda) \subset \boldsymbol{C}$, where $D(\lambda) \subset \boldsymbol{C}$ is $\boldsymbol{R}^{2}$ if $\lambda \notin \boldsymbol{Q}$. If $\lambda= \pm n / m \in \boldsymbol{Q}$ with $n, m \in \boldsymbol{N},\langle n, m\rangle=1$, then we have $D(\lambda)=\boldsymbol{R} \times[0,2 \pi m]$. Again we consider the Fubini-Study metric of $\boldsymbol{C} P(2)$. The metric induced by $\varphi_{(x, y)}$ on $\boldsymbol{C}$ is written as

$$
\mu^{2}(x, y)=\varphi_{(x, y)}^{*}\left(d s^{2}\right)=4 \frac{e^{2 \operatorname{Re}(z)}|x|^{2}+|\lambda|^{2}|y|^{2} e^{2 \operatorname{Re}(\lambda z)}+|\lambda-1|^{2}|x y|^{2} e^{2 \operatorname{Re}[(\lambda+1) z]}}{\left(1+|x|^{2} e^{2 \operatorname{Re}(z)}+|y|^{2} e^{2 \operatorname{Re}(\lambda z)}\right)^{2}}|d z|^{2} .
$$

The area form induced by $\varphi$ is then given by

$$
d \sigma(x, y)=4 \frac{\left[|x|^{2} e^{2 \operatorname{Re}(z)}+|y \lambda|^{2} e^{2 \operatorname{Re}(\lambda z)}+|x y|^{2}|\lambda-1|^{2} e^{2 \operatorname{Re}[(\lambda+1) z]}\right]}{\left(1+|x|^{2} e^{2 \operatorname{Re}(z)}+|y|^{2} e^{2 \operatorname{Re}(\lambda z)}\right)^{2}} d V,
$$


which yields

$$
d \sigma(x, y)=4 \frac{\left[|x|^{2} e^{2 u}+|\lambda y|^{2} e^{2(\alpha u-\beta v)}+|x y|^{2}|\lambda-1|^{2} e^{2(\alpha u-\beta v+u)}\right]}{\left(1+|x|^{2} e^{2 u}+|y|^{2} e^{2(\alpha u-\beta v)}\right)^{2}} d u d v,
$$

where we write $\lambda=\alpha+\sqrt{-1} \beta$ and $z=u+\sqrt{-1} v$. Performing the change of variables $s=e^{2 u}, t=e^{-2 v}$, we obtain

$$
d \sigma(x, y)=\frac{|x|^{2} t^{-1}+|y|^{2}|\lambda|^{2} s^{\alpha-1} t^{\beta-1}+|x|^{2}|y|^{2}|\lambda-1|^{2} s^{\alpha} t^{\beta-1}}{\left(1+|x|^{2} s+|y|^{2} s^{\alpha} t^{\beta}\right)^{2}} d s d t .
$$

Proposition 1. If $\boldsymbol{\lambda} \in \boldsymbol{C} \backslash \boldsymbol{Q}$ then $\operatorname{Vol}_{X}\left(x_{0}, y_{0}\right)=+\infty$, provided that $x_{0}, y_{0} \neq 0$ in $\boldsymbol{C}^{2}$. Moreover, if $\lambda \in \boldsymbol{Q}$, then $\operatorname{Vol}_{X}$ is integrable in $\boldsymbol{C}^{2}$.

Proof. If $\lambda \in \boldsymbol{Q}$, then the volume function is given by

$$
\operatorname{Vol}_{X}\left(x_{0}, y_{0}\right)=\int_{t=e^{-4 \pi m}}^{t=1} \int_{s=0}^{s=+\infty} d \sigma\left(x_{0}, y_{0}\right) .
$$

Now we recall the fact that $\int_{0}^{+\infty} 1 /\left(1+u^{\nu}\right) d u$ converges for $v>1$. Note that, for $\lambda<1$, the term $u^{v}$ is given by $\left(\left|x_{0}\right|^{2} u\right)^{2}$ in the denominator. For $\lambda \in(1,+\infty)$, it is given by $\left(\left|y_{0}\right|^{2} u^{\lambda}\right)^{2}$. The case $\lambda=1$ gives $(\lambda-1)^{2}\left|x_{0} y_{0}\right|^{2} u^{\lambda}=0$ in the numerator, for which we take $v$ given by $\left(\left|x_{0}\right|^{2} u\right)^{2}$ for instance. Thus we conclude that the volume is integrable in the case $\lambda \in Q$.

Assume now that $\lambda \in \boldsymbol{C} \backslash \boldsymbol{Q}$. In this case, we consider the integral

$$
I(\alpha, \beta):=\iint_{R^{2}} \frac{\left|x_{0}\right|^{2} t^{-1}}{\left(1+\left|x_{0}\right|^{2} s+\left|y_{0}\right|^{2} s^{\alpha} t^{\beta}\right)^{2}} d s d t
$$

and use the following fact, which completes the proof of the proposition.

Claim 2. The value of the integral $\int_{0}^{+\infty} t^{-1} /\left(1+t^{\beta}\right)^{2} d t$ is infinite for any $\beta \in \boldsymbol{R}$.

Indeed, assume that $\beta \geq 0$. For $0 \leq t \leq 1$, we have $1+t^{\beta} \leq 2$ and therefore $1 /\left(1+t^{\beta}\right)^{2} \geq 1 / 4$, which yields $\int_{0}^{1}\left[t^{-1} /\left(1+t^{\beta}\right)^{2}\right] d t \geq(1 / 4) \int_{0}^{1} d t / t=+\infty$. Assume now that $\beta=-\gamma<0$. Then

$$
\frac{t^{-1}}{\left(1+t^{-\gamma}\right)^{2}}=\frac{1}{t+2 t^{1-\gamma}+t^{1-2 \gamma}}>\frac{1}{t+K}
$$

for some constant $K>0$ and sufficiently large $t>M$. Hence $\int_{0}^{+\infty} t^{-1} /\left(1+t^{\beta}\right)^{2} d t \geq$ $\int_{M}^{+\infty} 1 /(t+K) d t=+\infty$.

LEMMA 2. Let $X$ be a meromorphic vector field in a complex manifold $\bar{M}$ equipped with a $C^{2}$ Riemannian metric which is Hermitian along the leaves of the induced foliation $\mathcal{F}(X)$. Assume that $\operatorname{Vol}_{X}(x)<+\infty$ for a certain $x \in M \backslash \operatorname{sing} \mathcal{F}(X)$. Then the leaf $L=L_{x}$ is closed outside $\operatorname{sing} \mathcal{F}(X)$.

Proof. Fix a point $p \in \bar{L} \backslash \operatorname{sing} \mathcal{F}(X)$. By the flow-box theorem there exists a complex diffeomorphism $\varphi$ from a small neighborhood $W$ of $p$ onto a polydisc $\Delta$ in $C^{n}$, which takes $\left.\mathcal{F}(X)\right|_{W}$ into the horizontal foliation $\mathcal{H}$. The map $\varphi$ is locally Lipschitz so that the sum of the 
volumes of the plaques of $L$ in $W$ is finite if and only if there are only finitely many plaques of $\mathcal{H}$ in $\Delta$ that correspond to the intersection $L \cap W$. This implies that $L \cap W$ consists of finitely many plaques and therefore $p \in L$. This shows that $L$ is closed in $\bar{M} \backslash \operatorname{sing} \mathcal{F}(X)$.

A consequence of this lemma is the following

Proposition 2. Let $X$ be a polynomial vector field on $\boldsymbol{C}^{2}$ such that (i) the singularities of $\mathcal{F}(X)$ are hyperbolic, and (ii) there exists a nonsingular orbit $\mathcal{O}_{x} \subset L_{x}$ which has finite volume for the Fubini-Study metric in duced on $\boldsymbol{C}^{2}$. Then $\bar{L}_{x}$ is an algebraic curve.

Proof. The orbit $\mathcal{O}_{x}$ is closed in $\boldsymbol{C}^{2} \backslash \operatorname{sing} \mathcal{F}(X)$ by Lemma 2. If there exists $p \in$ $\overline{\mathcal{O}}_{x} \cap \operatorname{sing} \mathcal{F}(X)$, then, by the local structure of hyperbolic singularities in the complex case, it follows that $\overline{\mathcal{O}}_{x}$ must be locally contained in the two separatrices of $X$ at $p$. Thus $\overline{\mathcal{O}}_{x}$ is analytic of dimension one in $\boldsymbol{C}^{2}$.

Now we consider $\mathcal{F}(X)$ in a neighborhood of the line $L_{\infty}=\boldsymbol{C P}(2) \backslash \boldsymbol{C}^{2}$. Then there are two possibilities:

Case 1. $L_{\infty}$ is not invariant by $\mathcal{F}(X)$. In this case, given any point $p \in L_{\infty} \backslash \operatorname{sing} \mathcal{F}(X)$, by the flow-box theorem the leaf $L_{x}$ is locally closed around $p$ and therefore analytic around $p$. Let now $p \in L_{\infty} \cap \operatorname{sing} \mathcal{F}(X) \cap \overline{\mathcal{O}}_{x}$. Since $p$ is hyperbolic and $L_{x}$ is closed in $\boldsymbol{C}^{2}$, it follows again that $\bar{L}_{x}$ is contained in the set of separatrices of $\mathcal{F}(X)$ at $p$. Thus $\bar{L}_{x}$ is analytic in $\boldsymbol{C P}(2)$.

Case 2. $L_{\infty}$ is invariant by $\mathcal{F}(X)$. By the maximum principle, $\mathcal{O}_{x}$ accumulates $L_{\infty}$ and, since it is invariant, it follows that $L_{x}$ accumulates some singularity $p \in \operatorname{sing} \mathcal{F}(X) \cap L_{\infty}$. As above, the hyperbolic type of $p$ implies that $\bar{L}_{x}$ is contained in the separatrix transverse to $L_{\infty}$ at $p$. Thus again we conclude that $\bar{L}_{x}$ is analytic of dimension one in $\boldsymbol{C P}(2)$.

Consequently, it is implied by Remmert-Stein theorem [16] that $\bar{L}_{x}=\overline{\mathcal{O}}_{x}$ is analytic in $\boldsymbol{C P}(2)$ and by Chow theorem [16] it is an algebraic curve.

Proof OF THEOREM 1. The proof is now reduced to a mimic of the original proof of Mattei-Moussu theorem ([22]) if one remarks the following: (1) Almost every leaf has finite Euclidean volume and is therefore closed outside the origin $0 \in \boldsymbol{C}^{2}$ (Lemma 2). (2) All virtual holonomy groups arising in the reduction process of $\operatorname{sing} \mathcal{F}(X)$ are finite and therefore each one is conjugate to a cyclic group of rational rotations (Lemma 1).

Proof of TheOREM 2. If $L_{\infty}=\boldsymbol{C P}(2) \backslash \boldsymbol{C}^{2}$ is not invariant by the foliation, then the leaves of $\mathcal{F}(X)$ are also closed in $C P(2) \backslash \operatorname{sing} \mathcal{F}(X)$. As above, this implies that the leaves are contained in algebraic curves. By Darboux theorem ([20]) the foliation admits a rational first integral. We may therefore assume that $L_{\infty}$ is invariant and by Theorem 6 conclude that $\mathcal{F}(X)$ has some separatrix $\Gamma$ transverse to $L_{\infty}$, with $\Gamma \cap L_{\infty}=p \in \operatorname{sing} \mathcal{F}(X)$. After performing the reduction of singularities for $\left.\mathcal{F}(X)\right|_{L_{\infty}}$, we then obtain a strict transform $\tilde{\Gamma}$ transverse to the divisor $D=\bigcup_{j=1}^{m} D_{j}$, which cuts $D$ at a singularity $\tilde{p}=\tilde{\Gamma} \cap D=\tilde{\Gamma} \cap \boldsymbol{P}_{j_{o}}$. The local holonomy associated to $\tilde{\Gamma}^{*}=\tilde{\Gamma} \backslash\{\tilde{p}\}$ has finite pseudo-orbits and is therefore finite. By a result of Mattei-Moussu ([22]), $\tilde{\mathcal{F}}$ is linearizable around $\tilde{p}$ as $n x d y+m y d x=0, n, m \in N$ and $\tilde{\Gamma}:\{x=0\}, \boldsymbol{P}_{j_{o}}:\{y=0\}$. The virtual holonomy group associated to a small transverse 
section disk $\Sigma_{j}, \Sigma_{j} \cap \boldsymbol{P}_{j}=q_{j} \notin \operatorname{sing} \tilde{\mathcal{F}}(X)$, has discrete pseudo-orbits outside the origin $q_{j}$ and hence is solvable ([26]). This holds for any component $\boldsymbol{P}_{j}$ of the divisor $D$.

Since $\tilde{p}$ is of type $d\left(x^{m} y^{n}\right)=0$, we may consider the adjunction of the holonomy group $\operatorname{Hol}\left(\tilde{\mathcal{F}}(X), \boldsymbol{P}_{j_{o}}\right)$ to the local holonomy $h_{o}$ of $\tilde{\Gamma}^{*}$ and obtain a subgroup $\mathcal{D}_{q_{*}} \operatorname{Hol}\left(\tilde{\mathcal{F}}(X), \boldsymbol{P}_{j_{o}}\right) *$ $\left\langle h_{o}\right\rangle \subset \hat{\operatorname{Hol}}(\tilde{\mathcal{F}}(X), \Sigma)$, where $\Sigma$ is a transverse section $\{y=$ cte $\}$ at a point in $\tilde{\Gamma}^{*}$, and $\hat{\operatorname{Hol}}(\tilde{\mathcal{F}}(X), \Sigma)$ denotes the virtual holonomy at this section. This adjunction group has also finite pseudo-orbits (as well as the virtual holonomy $\hat{\operatorname{Hol}}(\tilde{\mathcal{F}}(X), \Sigma)$ ) and is therefore finite. Thus, using the construction given in Section 2 for the group $\mathcal{D}_{q_{*}} \operatorname{Hol}\left(\tilde{\mathcal{F}}(X), \boldsymbol{P}_{j_{o}}\right) *\left\langle h_{o}\right\rangle$, we conclude that $\operatorname{Hol}\left(\tilde{\mathcal{F}}(X), \boldsymbol{P}_{j_{o}}\right)$ is also finite (recall the construction in Section 2: In fact, each element $g \in \operatorname{Hol}\left(\tilde{\mathcal{F}}(X), \boldsymbol{P}_{j_{o}}\right)$ induces an element $g^{\mathcal{D}_{q}}$ in the adjunction group, which is a periodic rotation. Using the explicit formulae for $g^{\mathcal{D}_{q}}$ given by the possible cases (i.e., $\operatorname{Hol}\left(\tilde{\mathcal{F}}(X), \boldsymbol{P}_{j_{o}}\right)$ is abelian or solvable, nonabelian isomorphic to a finite ramified covering of a group of homographies, and in this last case $g^{\mathcal{D}_{q}}$ is also a finite ramified covering of a homography and cannot be finite) we see that $g$ is also a rational rotation. Alternatively, one may prove that $\operatorname{Hol}\left(\tilde{\mathcal{F}}(X), \boldsymbol{P}_{j_{o}}\right)$ has finite pseudo-orbits, using the fact that the Dulac correspondence associated to the corner $\tilde{p}$ is a finite-to-finite correspondence (see Section 2).

At this stage we know that $\tilde{\mathcal{F}}$ has a holomorphic first integral in a neighborhood of $\boldsymbol{P}_{j_{o}} \cup \tilde{\Gamma}$. Given any corner $p_{j}=\boldsymbol{P}_{j_{o}} \cap \boldsymbol{P}_{j}$, we may iterate the above process: Indeed, $p_{j}$ is necessarily of type $d\left(x^{n} y^{m}\right)=0, n, m \in N$ ([22]) and, as above, we may perform the adjunction of the holonomy of $\boldsymbol{P}_{j}$ to the holonomy of $\boldsymbol{P}_{j_{o}}$. This gives a new group that by its turn will constitute a group $\mathcal{D}_{\tilde{p}} *\left(\mathcal{D}_{p_{j}} * \operatorname{Hol}\left(\tilde{\mathcal{F}}(X), \boldsymbol{P}_{j}\right) * \operatorname{Hol}\left(\tilde{\mathcal{F}}(X), \boldsymbol{P}_{j_{o}}\right)\right) *\left\langle h_{o}\right\rangle \subset \hat{\operatorname{Hol}}(\tilde{\mathcal{F}}(X), \Sigma)$. As above, the new adjunction group is finite and therefore $\operatorname{Hol}\left(\tilde{\mathcal{F}}(X), \boldsymbol{P}_{j}\right)$ must be finite. Since the group $\mathcal{D}_{\tilde{p}} *\left(\mathcal{D}_{p_{j}} * \operatorname{Hol}\left(\tilde{\mathcal{F}}(X), \boldsymbol{P}_{j}\right) * \operatorname{Hol}\left(\tilde{\mathcal{F}}(X), \boldsymbol{P}_{j_{o}}\right)\right) *\left\langle h_{o}\right\rangle \subset \hat{\operatorname{Hol}}(\tilde{\mathcal{F}}(X), \Sigma)$ is finite, we may construct a holomorphic first integral for $\tilde{\mathcal{F}}(X)$ in a neighborhood of $\tilde{\Gamma} \cup \boldsymbol{P}_{j_{o}} \cup \boldsymbol{P}_{j}$. A standard induction argument then shows that we may construct a holomorphic first integral for $\tilde{\mathcal{F}}(X)$ in a neighborhood of the invariant part of the divisor $D=\bigcup_{j} \boldsymbol{P}_{j}$. Since, by hypothesis, the complement of the invariant part of the resolution divisor of $\operatorname{sing} \mathcal{F}(X) \cap L_{\infty}$ is pseudo-convex, we may extend this function to a rational function ([36]) which blows-down to a rational first integral $R$ for $\mathcal{F}(X)$ in $\boldsymbol{C P}(2)$ (see [9]).

Proof of THEOREM 3. The proof for Theorem 2 also works here, since we only need to use the facts that $\hat{\operatorname{Hol}}(\tilde{\mathcal{F}}(X), \Sigma)$ is finite and $\hat{\operatorname{Hol}}\left(\tilde{\mathcal{F}}(X), \boldsymbol{P}_{j}\right)$ is solvable with discrete pseudo-orbits off the origin, which also hold provided that the leaves of $\left.\mathcal{F}\right|_{C^{2}}$ are closed in $C^{2} \backslash \operatorname{sing} \mathcal{F}(X)$.

3.1. Applications. In this paragraph we give some applications of the preceding results.

PROPOSITION 3. Let $\mathcal{F}$ be a foliation with rational first integral on $\boldsymbol{C P}(2)$ and $\boldsymbol{C}^{2} \subset$ $\boldsymbol{C P}(2)$ an affine space such that all singularities of $\mathcal{F}$ in $L_{\infty}$ are of rational type. Let $\mathcal{F}_{1}$ be any foliation on $\boldsymbol{C P}(2)$ which is topologically conjugate to $\mathcal{F}$ on $\boldsymbol{C}^{2}$ and have also rational type singularities in $L_{\infty}$. Then $\mathcal{F}_{1}$ admits a rational first integral. 
ProOF. First we notice that by topological equivalence the leaves of $\mathcal{F}_{1}$ are closed in $C^{2} \backslash \operatorname{sing} \mathcal{F}_{1}$. Since the singularities of $\mathcal{F}_{1}$ in $L_{\infty}$ are also rational, it follows from Theorem 3 that $\mathcal{F}_{1}$ admits a rational first integral.

The hypothesis on $\operatorname{sing} \mathcal{F} \cap L_{\infty}$ may not be omitted as illustrated by the following example.

EXAMPLE 3. Let $\mathcal{F}: d y-y d x=0$. Then $\mathcal{F}$ admits no rational first integral, for it admits the (primitive) algebroide first integral $y e^{-x}$. In particular, its leaves are closed in $\boldsymbol{C}^{2}$. On the other hand, the homeomorphism $\phi: \boldsymbol{C}^{2} \rightarrow \boldsymbol{C}^{2}$ defined by $\phi(x, y)=\left(x, x e^{-x} y\right)$ conjugates $\mathcal{F}$ to the radial foliation $\mathcal{R}: x d y-y d x=0$, which has the rational first integral $R=x / y$. We have $\operatorname{sing} \mathcal{R} \cap L_{\infty}=\emptyset$ with $L_{\infty}$ transverse to $\mathcal{R}$, but the same does not hold for $\mathcal{F}$; there is a saddle-node singularity $\dot{u}=u^{2}, \dot{v}=v(u-1)$ in $L_{\infty}$.

In what follows we refer to [34] and [37] for the notion of generic type of the orbits of a holomorphic flow on a Stein surface.

Proposition 4. Let $X$ be a complete polynomial vector field in $\boldsymbol{C}^{2}$. Assume that

(i) the generic type of the orbit of $X$ is $C^{*}$, and

(ii) the singularities of $\mathcal{F}(X)$ in $L_{\infty}$ are of rational type.

Then $\mathcal{F}(X)$ admits a rational first integral and one of the following occurs:

(a) $X$ has no dicritical singularity in $\boldsymbol{C}^{2}$ and there exists a (non-constant) polynomial first integral $F: \boldsymbol{C}^{2} \rightarrow \boldsymbol{C}$.

(b) $X$ has a dicritical singularity in $C^{2}$ and there exists an entire automorphism $\psi \in$ $\operatorname{Aut}\left(\boldsymbol{C}^{2}\right)$, which linearizes $X$, that is, $\psi_{*} X=n x(\partial / \partial x)+m y(\partial / \partial y), n, m \in N$. In particular, there exists only one dicritical singularity in $C^{2}$.

PROOF. According to [37], there exists a meromorphic first integral on $\boldsymbol{C P}(2)$. Since $\operatorname{sing} \mathcal{F}(X) \cap L_{\infty}$ consists of rational singularities, Theorem 6 assures the existence of a rational first integral. Using now [11], we find (a) and (b) as the only possibilities.

COROLlaRY 1. Let $\mathcal{F}_{1}$ and $\mathcal{F}_{2}$ be germs of holomorphic foliations at $0 \in \boldsymbol{C}^{2}$ such that there exists a germ of measure isomorphism $T: C^{2}, 0 \rightarrow C^{2}, 0$ conjugating $\mathcal{F}_{1}$ to $\mathcal{F}_{2}$. Then $\mathcal{F}_{1}$ admits a holomorphic first integral if and only if $\mathcal{F}_{2}$ admits a holomorphic first integral.

PROOF. In fact, a measure isomorphism conjugating the foliations preserves the volume functions of the foliations, so that it suffices to use Theorem 1.

4. Sub-exponential growth. Our goal here is to show another approach to the study of the behavior of foliations on $\boldsymbol{C P}(2)$, with geometric features. We refer to [28] and [29] for the notion of sub-exponential growth of a leaf.

Let $\mathcal{F}$ be a foliation with hyperbolic singularities on $\boldsymbol{C P}(2)$, and $g$ a $C^{\infty}$ Riemannian metric on $\boldsymbol{C P}(2)$, which is Hermitian along the leaves of $\mathcal{F}$. Suppose that $\operatorname{sing} \mathcal{F}=$ $\left\{p_{1}, \ldots, p_{r}\right\}$, and for each $j \in\{1, \ldots, r\}$ choose a small closed ball centered at $p_{j}$, say, 
$\overline{\boldsymbol{B}}\left(p_{j}\right) \ni p_{j}$. Since $p_{j}$ is hyperbolic, $\mathcal{F}$ is transverse to the spheres $\partial \overline{\boldsymbol{B}}\left(p_{j}\right)$ and hence we may consider the double of $\mathcal{F}$ as in [2] and [34]. This is a $C^{\infty}$ regular codimension-two real foliation $\mathcal{F}_{d}$ on a compact real 4-manifold $\bar{M}_{d}$, which contains two copies of the foliated pair

$$
\left(\boldsymbol{C P}(2) \backslash \bigcup_{j=1}^{r} \overline{\boldsymbol{B}}\left(p_{j}\right),\left.\mathcal{F}\right|_{\boldsymbol{C} P(2) \backslash \bigcup_{j=1}^{r} \overline{\boldsymbol{B}}\left(p_{j}\right)}\right) .
$$

The leaves of $\mathcal{F}_{d}$ have also natural structures of Riemann surfaces and the metric $g$ induces a $C^{\infty}$ Riemannian metric $g_{d}$ in $\bar{M}_{d}$, that can be chosen to be Hermitian along the leaves of $\mathcal{F}_{d}$. The corresponding leaves $L_{d}$ of $\mathcal{F}_{d}$ have sub-exponential growth for $g_{d}$ so that, using Theorem 4.1 of [29], we obtain

Proposition 5. Let $\mathcal{F}, g$ be as in Theorem 4. Then there exists a non-trivial holonomy invariant transverse measure $\mu$ for $\mathcal{F}_{d}$, which is finite on compact sets and whose support is contained in the closure $\bar{L}_{d}$ of some leaf $L_{d}$ of $\mathcal{F}_{d}$.

Now we take $K=\operatorname{supp}(\mu) \subset \bar{M}_{d}$ the support of $\mu$. We have a compact $\mathcal{F}$-saturated non-empty set. According to [6], we know that a non-trivial minimal set on $\boldsymbol{C P}(2)$ cannot support an invariant transverse measure. Hence one may conclude.

Lemma 3. $K \cap \partial \boldsymbol{B}\left(p_{j}\right) \neq \emptyset$ for some $j \in\{1, \ldots, r\}$.

As in [2] and [34], this implies that if $L$ is a leaf of $\mathcal{F}$ such that $L \backslash \bigcup_{j=1}^{r} \overline{\boldsymbol{B}}\left(p_{j}\right) \subset K$, then $\bar{L}=L \cup\left\{p_{j_{1}}, \ldots, p_{j_{l}}\right\}$ for some $p_{j_{v}} \in \operatorname{sing} \mathcal{F}$, and therefore by Remmert-Stein theorem and Chow theorem [16], $\bar{L}$ is an algebraic curve on $\boldsymbol{C P}(2)$. Hence we obtain.

Lemma 4. Let $\mathcal{F}, g$ be as in Theorem 4 . Then $\mathcal{F}$ has some algebraic invariant curve $S \subset \boldsymbol{C P}(2)$.

We consider the algebraic leaf $S \subset \boldsymbol{C P}(2)$, given by Lemma 4, and take a point $q \in$ $S \backslash \operatorname{sing} \mathcal{F}$, and a small transverse disk $\Sigma \subset \boldsymbol{C} P(2)$ with $\Sigma \cap S=\{q\}$. Thus we may consider the holonomy group $\operatorname{Hol}(\mathcal{F}, S, \Sigma)$ as a subgroup of $\operatorname{Diff}(\Sigma, q) \approx \operatorname{Diff}(\boldsymbol{C}, 0)([8])$.

LEMMA 5. $\operatorname{Hol}(\mathcal{F}, S, \Sigma)$ is a solvable group.

Proof. This is proved in [32]. The idea is as follows. Denote by $\Lambda$ the set of algebraic leaves of $\mathcal{F}$ in $\boldsymbol{C P}(2)$. We have $S \subset \Lambda$. According to [32] a compact total transverse section to $\mathcal{F}$ is a compact (real $C^{\infty}$ ) submanifold $N \subset \boldsymbol{C} P(2) \backslash \operatorname{sing} \mathcal{F}$ (possibly with boundary), of dimension two, such that every leaf of $\mathcal{F}$ intersects the interior of $N$ and $\mathcal{F}$ is transverse to $N \backslash \partial N$ (see the Remark after Corollary 4.2 in page 340 of [29]). It is not difficult to prove that, as a consequence of the compactness of $C P(2)$ together with the maximum principle, the foliation $\mathcal{F}$ admits a compact total transverse section; indeed, $N$ is a finite union of closed disks whose interiors are transverse to $\mathcal{F}$ ([32]). Therefore, again according to the Remark in page 340 of [29], it follows that, for $M=C P(2) \backslash \Lambda$, the restriction $\left.\mathcal{F}\right|_{M}$ admits a non-trivial holonomy invariant transverse measure $\mu$. To get a contradiction assume that $\operatorname{Hol}(\mathcal{F}, S, \Sigma)$ is non-solvable. Any non-algebraic leaf $L_{o}$ must accumulate the point $q=\Sigma \cap S$ in $\Sigma$ and therefore, as it follows from [26], the leaf $L_{o}$ is dense in a neighborhood $V \subset \Sigma$ of $q$ 
and $\operatorname{Hol}(\mathcal{F}, S, \Sigma)$ has a dense set of hyperbolic periodic pseudo-orbits on $V$ (i.e., a dense set of hyperbolic fixed points). Given such a fixed point $z_{o} \in V$ we consider the word $f \in$ $\operatorname{Hol}(\mathcal{F}, S, \Sigma)$ such that $f\left(z_{o}\right)=z_{o}$, with $\left|f^{\prime}\left(z_{o}\right)\right| \neq 1$. This map $f$ may be linearized in a small disk $D\left(z_{o}, \varepsilon\right) \subset V$ as $f(z)=\lambda z$ with $\lambda \in C^{*} \backslash S^{1}$. Notice that this local map corresponds to the local holonomy map of some cycle $\gamma_{z_{o}}$ in the leaf $L_{z_{o}} \subset M$ and therefore, since $\mu$ is $\operatorname{Hol}\left(\left.\mathcal{F}\right|_{M}, L_{z_{o}}\right)$-invariant, it follows that $\left.\mu\right|_{D\left(z_{o}, \varepsilon\right)}$ is the Dirac measure with atom at $z_{o}$. In particular, $\operatorname{supp}(\mu) \cap D\left(z_{o}, \varepsilon\right)=\left\{z_{o}\right\}$. On the other hand, since $L_{x}$ is dense in $V$ we have $\overline{L_{x}} \cap D\left(z_{o}, \varepsilon\right)=D\left(z_{o}, \varepsilon\right)$ which gives $\operatorname{supp}(\mu) \cap D\left(z_{o}, \varepsilon\right)=D\left(z_{o}, \varepsilon\right)$, contradiction. This contradiction proves Lemma 5 .

Proof of Theorem 4. We consider an affine chart $(x, y) \in \boldsymbol{C}^{2} \subset \boldsymbol{C P}(2)$, and a polynomial 1-form $\omega=P d x+Q d y$ with isolated singularities, which defines $\left.\mathcal{F}\right|_{C^{2}}$. Since $\operatorname{Hol}(\mathcal{F}, S, \Sigma)$ is solvable, and $\mathcal{F}$ has hyperbolic singularities, it follows from [31] that there exists a closed rational 1-form $\eta$ on $\boldsymbol{C P}(2)$, with simple poles, such that $d \omega=\eta \wedge \omega$. In particular, $\mathcal{F}$ is transversely affine outside some algebraic invariant set (given by $(\eta)_{\infty} \cap \boldsymbol{C}^{2}$ [31]). Since $\mathcal{F}$ has hyperbolic singularities, this implies that $\mathcal{F}$ is a Darboux (logarithmic) foliation ([33], [34]), and hence must be linear (because of the hyperbolic singularities [2]), as in Theorem 4.

5. Orbits having finite total curvature. Let $\psi: M \rightarrow \bar{M}=\boldsymbol{R}^{n}$ be a minimal isometric immersion of a two-dimensional real manifold $M$ into a $n$-dimensional Euclidean space $\boldsymbol{R}^{n}$. When $M$ is orientable, we have a natural conformal structure given by the isothermal coordinates, and consider a local conformal coordinate $z=u+\sqrt{-1} v: U \subset \boldsymbol{C} \rightarrow M$ on $M$ Since $\psi$ is conformal, the metric induced by $\psi$ is written as $d s^{2}=2 F|d z|^{2}$, where $F=|\varphi|^{2}$, and $\varphi=\left(\varphi_{1}, \ldots, \varphi_{n}\right): U \rightarrow \boldsymbol{C}^{n}$ defined by

$$
\varphi_{j}=\frac{\partial \psi_{j}}{\partial z}=\frac{1}{2}\left(\frac{\partial}{\partial u}-\sqrt{-1} \frac{\partial}{\partial v}\right)\left(\psi_{j}\right), \quad j=1,2, \ldots, n,
$$

is holomorphic. The fact that $\psi$ is conformal also implies $\varphi^{2}=\sum_{j=1}^{n} \varphi_{j}^{2}=0$. The LaplaceBeltrami operator induced by $\psi$ is given by

$$
\Delta=\frac{2}{F} \frac{\partial}{\partial z} \frac{\partial}{\partial \bar{z}}
$$

where

$$
\frac{\partial}{\partial z}:=\frac{1}{2}\left(\frac{\partial}{\partial u}-\sqrt{-1} \frac{\partial}{\partial v}\right)
$$

and $z=u+\sqrt{-1} v \in U \subset \boldsymbol{C}$. The Gaussian curvature of the isometric immersion is therefore given by

$$
K=-\frac{1}{F} \frac{\partial}{\partial z} \frac{\partial}{\partial \bar{z}} \log F=-\frac{1}{2} \Delta \log F
$$


which can be also written as

$$
K=-\left|\varphi \wedge \varphi^{\prime}\right|^{2} /|\varphi|^{6} .
$$

In particular, we see that $K \leq 0$, and (since $\varphi$ is holomorphic) either $K \equiv 0$ or the set $\{K=0\}$ is discrete. This allows us to define the total curvature of the immersion as $C(\psi)=\int_{M} K d \sigma^{2} \in[-\infty, 0]$, where $d \sigma^{2}$ is the induced area element in $M$. A well-known result due to Huber ([18]) asserts the following

THEOREM 7 ([18], [38]). Let $\psi: M \rightarrow \boldsymbol{R}^{n}, n \geq 3$, be a minimal immersion, which is complete and has finite total curvature. Then $M$ is conformally equivalent to a finitely punctured compact Riemann surface.

Subsequently, Osserman ([27]) showed that the Gauss map of such an embedding extends meromorphically to the ends of $M$. This result is one of the main tools in the study of complete finitely curved minimal immersions into Euclidean spaces. In this section we study this problem for the orbits of a vector field on a complex manifold: Let $X$ be a holomorphic vector field on a complex manifold $\bar{M}$ equipped with a Kaehlerian metric. The orbits of $X$ are complex immersed submanifolds of $\bar{M}$ so that the inclusion gives minimal isometric immersions $\psi: M \rightarrow \bar{M}$. Examples of this situation are $\bar{M}=\boldsymbol{C}^{n}$ with its Euclidean structure, and $\bar{M}=\boldsymbol{C} P(n)$ with the Fubini-Study metric.

EXAMPLE 4. Let $X_{\lambda}=x(\partial / \partial x)+\lambda y(\partial / \partial y)$ be a linear vector field in $\boldsymbol{C}^{2} \cong \boldsymbol{R}^{4}$. Then we have a local parametrization $\psi(z)=\left(x e^{z}, y e^{\lambda z}\right)$ for the orbit $M=\mathcal{O}_{(x, y)}$ and $d s^{2}=\left(|x|^{2} e^{2 \operatorname{Re}(z)}+|\lambda y|^{2} e^{2 \operatorname{Re}(\lambda z)}\right)|d z|^{2}$. We write $\lambda=\alpha+\sqrt{-1} \beta, z=u+\sqrt{-1} v$, and obtain

$$
d s^{2}=\left(|x|^{2} e^{2 u}+|y|^{2}\left(\alpha^{2}+\beta^{2}\right) e^{2(\alpha u-\beta v)}\right)\left(d u^{2}+d v^{2}\right) .
$$

Now we recall that for any $C^{2}$ real function $f$ we have

$$
\Delta \log f=\Delta f / f-|\operatorname{grad} f|^{2} / f^{2},
$$

where $\operatorname{grad} f=\left(f_{u}, f_{v}\right)$ is the gradient of $f$. Thus for $f=|x|^{2} e^{2 u}+|y|^{2}\left(\alpha^{2}+\beta^{2}\right) e^{2(\alpha u-\beta v)}$ we find

$$
\Delta f=4\left[|x|^{2} e^{2 u}+|y|^{2}\left(\alpha^{2}+\beta^{2}\right)^{2} e^{2(\alpha u-\beta v)}\right]
$$

and

$$
|\operatorname{grad} f|^{2}=4\left[|x|^{4} e^{4 u}+\left(\alpha^{2}+\beta^{2}\right)^{3}|y|^{4} e^{4(\alpha u-\beta v)}+2 \alpha|x|^{2}|y|^{2}\left(\alpha^{2}+\beta^{2}\right) e^{2(\alpha u-\beta v+u)}\right] .
$$

Therefore

$$
f \Delta f-|\operatorname{grad} f|^{2}=4|x|^{2}|y|^{2}\left(\alpha^{2}+\beta^{2}\right)\left[(\alpha-1)^{2}+\beta^{2}\right] e^{2(\alpha u-\beta v+u)},
$$

which gives

$$
K=-8|x|^{2}|y|^{2}\left(\alpha^{2}+\beta^{2}\right)\left[(\alpha-1)^{2}+\beta^{2}\right] e^{2(\alpha u-\beta v+u)} /\left(|x|^{2} e^{2 u}+|y|^{2} e^{2(\alpha u-\beta v)}\right)^{3} .
$$

PROPOSITION 6. For an orbit $\psi: M \rightarrow \boldsymbol{R}^{4}$ of a linear vector field $X_{\lambda}=x(\partial / \partial x)+$ $\lambda y(\partial / \partial y)$ in $\boldsymbol{C}^{2}$, which is not contained in one of the complex axis, we have the following:

(i) If $\lambda \in \boldsymbol{R} \backslash \boldsymbol{Q}$, then $C(\psi)=-\infty$. 
(ii) If $\lambda \in(\boldsymbol{C} \backslash \boldsymbol{R}) \cup \boldsymbol{Q}$, then $C(\psi)>-\infty$.

Proof. Indeed, we have

$C(\psi)=-8|x|^{2}|y|^{2}\left(\alpha^{2}+\beta^{2}\right)\left[(\alpha-1)^{2}+\beta^{2}\right] \iint_{M} \frac{e^{2((\alpha+1) u-\beta v)}}{\left(|x|^{2} e^{2 u}+|y|^{2}\left(\alpha^{2}+\beta^{2}\right) e^{2(\alpha u-\beta v)}\right)^{3}} d u d v$.

Hence, changing coordinates for $s=e^{2 u}, t=e^{-2 v}$, we obtain

$$
C(\psi)=-2|x|^{2}|y|^{2}\left(\alpha^{2}+\beta^{2}\right)\left[(\alpha-1)^{2}+\beta^{2}\right] \iint_{W} \frac{s^{\alpha} t^{\beta-1}}{\left(|x|^{2} s^{2}+\left(\alpha^{2}+\beta^{2}\right)|y|^{2} s^{\alpha} t^{\beta}\right)^{3}} d s d t
$$

for a suitable domain $W=W(\lambda) \subset \boldsymbol{R}_{+} \times \boldsymbol{R}_{+}$(see Example 2). This proves the proposition.

Theorem 5 is therefore a kind of converse of the above example. Next we show that Theorem 5 is not true if we omit the hyperbolicity hypothesis on the singularities.

EXAMPLE 5. Let $X=\partial / \partial x+y(\partial / \partial y)$ be a non-singular vector field in $\boldsymbol{C}^{2}$. The foliation $\mathcal{F}(X)$ on $\boldsymbol{C P}(2)$ has a saddle-node singularity on $L_{\infty}: u^{2}(\partial / \partial u)+v(u-1)(\partial / \partial v)$. The orbit with an initial point $(x, y)$ is given by $\varphi(z)=\left(z+x, y e^{z}\right), z \in \boldsymbol{C}$, so that $d s^{2}=$ $\left(1+|y|^{2} e^{2 \operatorname{Re}(z)}\right)|d z|^{2}$ and therefore

$$
K=-8|y|^{2} e^{2 \operatorname{Re}(z)} /\left(1+|y|^{2} e^{2 \operatorname{Re}(z)}\right)^{3} .
$$

The foliation has the first integral $f=y e^{-x}$, so that the leaves are closed in $C^{2}$. However the total curvature of any leaf $L \not \subset(y=0)$ is $-\infty$.

In order to prove Theorem 5 we shall employ the following well-known lemma.

LEMMA 6. Let $L \subset \boldsymbol{C P}(2)$ be an orbit of $X$ such that the total curvature $C(L)$ of $L$ is zero. Then $\bar{L}$ is a straight line in $\boldsymbol{C P}(2)$.

Proof. We use the fact that $K=-\left|\varphi \wedge \varphi^{\prime}\right|^{2} /|\varphi|^{6}$ to conclude that $\varphi(z) \wedge \varphi^{\prime}(z) \equiv 0$ and therefore $\varphi^{\prime}(z)=\lambda(z) \varphi(z)$. Hence, by complex integration, we obtain

$$
\varphi(z)=\exp \left(\int_{o}^{z} \lambda(z) d z\right) w
$$

for some holomorphic function $\lambda(z)$ and some complex vector $w \in \boldsymbol{C}^{n}$. This implies that $\psi(L) \subset \boldsymbol{R}^{n}$ is contained in a complex line.

DEFINITION 3. A flat orbit is the one whose curvature is identically zero.

COROLLARY 2. Assume that $X$ is a polynomial vector field on $\boldsymbol{C}^{2}$ and has infinitely many flat orbits. Then, up to some affine change of coordinates, we have

(i) $X=\lambda(\partial / \partial x)$,

(ii) $X=\lambda(x(\partial / \partial x)+y(\partial / \partial y))$.

Proof. Since $X$ has infinitely many algebraic leaves, it follows from Darboux theorem [19] that it admits a rational first integral. According to Stein fatorization theorem ([14]), we may take such a primitive first integral, say, $R: \boldsymbol{C}^{2} \subset \boldsymbol{C} P(2) \rightarrow \boldsymbol{C P}(1)$. Thus if we write 
on $C^{2}, R=P / Q$, where $P$ and $Q$ are polynomials without nontrivial common factors, then the leaves of $\mathcal{F}$ are given by $\{\alpha P+\beta Q=0\}$ as $\alpha$ and $\beta$ vary on $\boldsymbol{C}$. Since infinitely many of these leaves are straight lines, it follows that $\max \{\operatorname{deg} P, \operatorname{deg} Q\}=1$. We have therefore two distinct cases:

(a) $0 \in \boldsymbol{C}^{2}$ is a dicritical singularity. In this case, $0 \in\{P=0\} \cap\{Q=0\}$ and we can find an affine change of coordinates which takes $\mathcal{F}$ into the foliation $d(x / y)=0$.

(b) There exists no dicritical singularity in $\boldsymbol{C}^{2}$. In this case, we conclude that $\{P=$ $0\} \cap\{Q=0\}=\varnothing$ and therefore we may find an affine change of coordinates which takes $\mathcal{F}$ into the foliation $d y=0$.

LEMMA 7. Let $M \subset \bar{M}$ be an orbit of $X$ having finite (negative) total curvature. Then $M$ is closed in $\bar{M} \backslash(\operatorname{sing} \mathcal{F}(X) \cup \Sigma)$, where $\Sigma \subset \bar{M}$ is the union of the flat orbits of $X$.

Proof. Indeed, let $p \in \bar{M} \backslash \operatorname{sing} \mathcal{F}(X)$ be an accumulation point of $M$ and denote by $L_{p}$ the leaf of $\mathcal{F}(X)$ containing $p$. Assume that $L_{p} \not \subset \Sigma$. Then $\{K=0\} \cap L_{p}$ is discrete and (since $M$ is invariant) we may assume that $K(p)<0$. By continuity, there exists a small neighborhood $V \ni p$ such that $K(q)<-\varepsilon$ for any $q \in V$ for some $\varepsilon>0$. By the flowbox theorem we may choose a neighborhood $p \in W \subset V$ where the flow of $X$ is trivial. This shows that either $p \in M$ or $M \cap W$ contains infinitely many plaques and therefore $C(M)=-\infty$. Since $C(M)$ is finite, $M$ is closed in $\bar{M} \backslash(\operatorname{sing} \mathcal{F}(X) \cup \Sigma)$.

The following is a local version of Theorem 5 .

PROPOSITION 7. Let $\mathcal{F}$ be a germ of holomorphic singular foliation at $0 \in \boldsymbol{C}^{2}$. Assume that for some neighborhood $V$ of 0 , the total curvature of the leaves is uniformly bounded, that there are no separatrices contained in straight lines in $\boldsymbol{C}^{2}$ and that the number of separatrices of $\mathcal{F}$ is finite. Then $\mathcal{F}$ admits a holomorphic first integral.

Proof. We denote by $\Sigma$ the union of flat leaves. According to Lemma 6, each leaf $L \subset$ $\Sigma$ is contained in a straight line in $\boldsymbol{C}^{2}$. Thus, according to our hypothesis on the separatrices, we conclude that $\Sigma=\emptyset$. Lemma 7 now implies that the leaves are closed outside the origin. Therefore, by Mattei-Moussu theorem ([22]), the foliation admits a holomorphic first integral.

Proof of THEOREM 5. First we consider the case where there are no affine orbits having total curvature equal to zero. In this case, according to Lemma 7 above, the leaves of $\mathcal{F}(X)$ are closed in $\boldsymbol{C}^{2}$ and therefore by Theorem 3 the foliation admits a rational first integral, which is absurd because it has hyperbolic singularities. Thus there are some orbits having Gaussian curvature identically zero. By Lemma 6, these orbits give some invariant complex line, say, $\boldsymbol{S} \subset \boldsymbol{C P}(2)$.

Now we consider the holonomy group of this line. By Lemma 7 we may assume that $\Sigma \subset \boldsymbol{C}^{2}$ is transversely discrete. Therefore, given a transverse disc $\boldsymbol{D}$ to $\mathcal{F}(X)$ centered at a point $o \in S \backslash \operatorname{sing} \mathcal{F}(X)$ and a generic point $z \in \boldsymbol{D}$, the pseudo-orbit of this point in the holonomy group $\operatorname{Hol}(\mathcal{F}(X), S, D)$ is discrete outside the origin. According to [5], since 
the holonomy group contains hyperbolic attractors (coming from the hyperbolic singularities of $\mathcal{F}(X)$ in $S$ ), this group must be abelian linearizable. This shows that $\mathcal{F}(X)$ is a linear logarithmic foliation on $\boldsymbol{C P}(2)$ ([5]). Using again the fact that the singularities are hyperbolic, we conclude that $\mathcal{F}(X)$ is linear in some affine chart ([2]).

Next we give a partial generalization of Theorem 5 .

Proposition 8. Let $X$ be a polynomial vector field on $C^{2}$ such that $\mathcal{F}(X)$ has only rational singularities. If some open orbit (by open orbit we mean one whose closure is not an algebraic curve in $\boldsymbol{C P}(2)$ ) of $X$ has finite total curvature, then $\mathcal{F}(X)$ is given by a closed rational 1-form on $\boldsymbol{C P}(2)$.

Proof. First we claim that $X$ has some algebraic orbits. Indeed, if otherwise the leaves of $\mathcal{F}(X)$ are closed in $C^{2} \backslash \operatorname{sing} \mathcal{F}(X)$ (Lemma 6) and therefore, by Theorem 3, $\mathcal{F}(X)$ has a rational first integral, which implies that all leaves are algebraic.

Let therefore $S \subset \boldsymbol{C P}(2)$ be an algebraic leaf of $\mathcal{F}(X)$ with $S \cap \boldsymbol{C}^{2} \neq \emptyset$. We may assume that $\mathcal{F}(X)$ has no rational first integral. Then, by Corollary 3, the set $\Sigma \subset \boldsymbol{C}^{2}$ of flat orbits consists of a finite number of orbits, so that given a small disc $\boldsymbol{D} \subset \boldsymbol{C}^{2}$ transverse to $\mathcal{F}$ with $\boldsymbol{D} \cap S=q \in S \backslash \operatorname{sing} \mathcal{F}(X)$, the virtual holonomy group $\hat{\operatorname{Hol}}(\mathcal{F}, \boldsymbol{D}, q)$ has discrete pseudo-orbits outside the origin $q \in \boldsymbol{D}$. Thus, this group is solvable ([26]).

Lemma 8. The 1-form $\omega=i_{X}(d V)$ (which is a polynomial 1-form with isolated singularities that defines $\mathcal{F}(X)$ in $C^{2}$ ) admits a rational logarithmic derivative $\eta$, with simple poles: $d \omega=\eta \wedge \omega, d \eta=0$. If all the virtual holonomy groups are abelian, then we may take $\eta$ with entire residues.

Proof. The 1-form $\eta$ is given by the main results in [8] and [33]. We explain here the main ideas, additional details can be found in [33], [9] and [11]. Let $\Lambda \subset D$ be an irreducible component of the divisor arising in the reduction of singularities for $\left.\mathcal{F}(X)\right|_{S}$. Let $\tilde{\omega}:=\pi^{*}(\omega)$. We first construct a transversely formal 1 -form $\eta=\hat{\eta}$ over the divisor $\Lambda$ (we refer to [11] and [17] for the notion of transversely formal $q$-form over a divisor on a projective surface): Recall that, according to [22] and [23], a nondegenerate nondicritical singularity always admits a formal integrating factor. Moreover, if $q$ is such a singularity, and $\hat{h}_{q}$ is such an integrating factor (defined as a formal expression at $q$ ), with respect to $\tilde{\omega}$ (that is, $\tilde{\omega} / \hat{h}_{q}$ is closed as a formal 1-form), then we can extend $\hat{h}_{q}$ as a transversely formal integrating factor for $\tilde{\omega}$, over a small disk $\boldsymbol{D}_{q} \subset \Lambda$ centered at $q$, using the resummation properties of the integrating factors along the separatrices for nondegenerate singularities. This is done by means of choosing a local system of coordinates $(x, y)$, centered at $q$ and such that $\Lambda:\{y=0\}$. Then, in these coordinates, we consider formal expressions $\hat{h}(x, y)=\sum_{j=0}^{+\infty} a_{j}(x) y^{j}$, where $a_{j}(x)$ are also formal positive series in the variable $x$. Now, if we impose that $\hat{h}$ is an integrating factor for $\tilde{\omega}$, then we obtain a differential equation which has a formal solution as remarked above, and the coefficients $a_{j}(x)$ are in fact analytic functions of $x$, in a fixed small disk centered at the origin, which is a consequence of Briot-Bouquet theorem type argument ([11]). 
Now we proceed as follows. First we assume that $\operatorname{Hol}(\Lambda)$ is abelian. According to [33], [9] and [11], there exists a transversely formal integrating factor $\hat{h}$ for $\tilde{\omega}$, defined over the open curve $\Lambda_{o}=\Lambda \backslash \operatorname{sing}(\tilde{\mathcal{F}}(X))$. We show that $\hat{h}$ may extend formally to $\operatorname{sing} \mathcal{F}(X) \cap \Lambda$. Indeed, given a (nondegenerate) singularity $q \in \Lambda$, we have the following three possibilities:

(1) If $\tilde{\omega}$ at $q$ is formally linearizable with a holomorphic local first integral. In this case, according to [22], $\tilde{\omega}$ admits a holomorphic first integral in a neighborhood of $q$, and therefore we may assume that $\hat{h}_{q}$ is in fact holomorphic in a neighborhood of $q$. Thus $\hat{h}$ extends to $q$, since it is already defined over the separatrix through $q$ tangent to $\Lambda_{o}$. In fact, we can find analytic coordinates $(x, y)$ centered at $q$ such that $\{y=0\}$ corresponds to $\Lambda$, and $\mathcal{F}$ is given in these coordinates by $n x d y+m y d x=0$, with $n, m \in \boldsymbol{Z}_{+},\langle n, m\rangle=1$. We take $\hat{h}_{o}=x y g$, where $g$ is the meromorphic function defined by $\tilde{\omega}(x, y)=g(n x d y+m y d x)$. Then we have $d\left(\tilde{\omega} / \hat{h}_{o}\right)=0$.

Now, the fact that $d(\tilde{\omega} / \hat{h})=0$, outside $\{x=0\}$, implies that $d\left(\hat{h}_{o} / \hat{h}\right) \wedge \tilde{\omega}=0$. Thus $\hat{f}=\hat{h}_{o} / \hat{h}$ is a meromorphic first integral for $\mathcal{F}$ along $\{x \neq 0\}$ and $\{y=0\}$. Since $q$ is nondicritical, it follows that either $\hat{f}$ is constant or we have $\hat{f}=\hat{\varphi}\left(\hat{f}_{o}\right)$ for some holomorphic one variable function $\varphi \in \boldsymbol{C}\{z\}$, where $\hat{f}_{o}=x^{m} y^{n}$ is a primitive holomorphic formal first integral for $\mathcal{F}$ at $q$. Therefore we have $\hat{f}=\varphi\left(x^{m} y^{n}\right)$. Since $\hat{f}$ is holomorphic formal along $\{y=0\} \subset \Lambda$ except at $q$, and $y=0$ implies $x^{m} y^{n}=0$, it follows that $\hat{\varphi}$ is holomorphic and therefore $\hat{f}$ extends holomorphically as $\varphi\left(\hat{f}_{o}\right)$ to $q$. This shows that $\hat{h}$ extends in a transversely formal way to $q$ in the case $\hat{f}$ is nonconstant. If $\hat{f}$ is constant, then the extension of $\hat{h}$ to $q$ is immediate.

(2) $\tilde{\omega}$ at $q$ is formally linearizable but admits no formal holomorphic first integral. In this case we claim that $\hat{h}$ extends to $q$ as a transversely formal integrating factor. Indeed, first we observe that $\tilde{\omega}$ admits no transversely formal first integral defined over a punctured disk $D \backslash\{q\}$ for a disk $D \subset \Lambda$. This is a consequence of [22] and [23] (see also [9]). On the other hand, the formal linearization of $\tilde{\omega}$ at $q$ gives a transversely formal integrating factor $\hat{h}_{1}$ for $\tilde{\omega}$ defined over a disk $D_{q} \subset \Lambda$ and centered at $q$. The quotient $\hat{f}:=\hat{h} / \hat{h}_{1}$ of two transversely formal integrating factors for $\tilde{\omega}$ over the punctured disk $D_{q} \backslash\{q\}$ in $\Lambda$ is a transversely formal first integral for $\tilde{\omega}$ and therefore it must be constant. This proves the extension of $\hat{h}$.

(3) If $\tilde{\omega}$ at $q$ is a resonant singularity but not formally linearizable (see [23]), then we have $k x d y+l y d x+$ h.o.t. $=0$ and $k, l \in N,\langle k, l\rangle=1$. It follows that $\hat{h}_{o} / \hat{h}$ is a formal meromorphic first integral for $\tilde{\omega}$ at $q$. But, since $\tilde{\omega}$ at $q$ is supposed to be nonlinearizable, it follows that no transversely formal first integral exists and therefore $\hat{h}_{o}=$ cte $\hat{h}$, so that $\hat{h}$ extends formally to $q$.

Now we assume that $\operatorname{Hol}(\Lambda)$ is solvable nonabelian. Using the techniques of [11] and [34], it is not difficult to obtain a transversely formal closed meromorphic 1-form $\hat{\eta}$, defined over $\Lambda_{o}$, which satisfies $d \tilde{\omega}=\hat{\eta} \wedge \tilde{\omega}$. Moreover, according to [21], we have a formal embed$\operatorname{ding} \operatorname{Hol}(\Lambda) \subset \boldsymbol{H}_{k}$. The construction of the 1 -form $\hat{\eta}$ gives $\operatorname{Res}_{\Lambda_{o}} \hat{\eta}=k+1+l$, where $l$ is the order of $\Lambda$ as a zero of the 1 -form $\tilde{\omega}$ ([8], [33]).

Fix a singularity $q \in \Lambda$. Once again we have the following three possibilities: 
(1) $\tilde{\omega}$ at $q$ is formally linearizable and admits a formal holomorphic first integral. In this case the extension follows as above.

(2) $\tilde{\omega}$ at $q$ is formally linearizable and does not admit a formal holomorphic first integral. The formal linearization gives a transversely formal integrating factor $\hat{h}_{1}$ defined over some disk $D_{q} \subset \Lambda$ centered at $q$. Define $\hat{\eta}_{1}=d \hat{h}_{1} / \hat{h}_{1}$. The difference $\hat{\Omega}:=\hat{\eta}-\hat{\eta}_{1}$ is a transversely formal closed 1-form defined over the punctured disk $D_{q} \backslash\{q\}$ and satisfying $\hat{\Omega} \wedge \tilde{\omega}=0$. Since $\tilde{\omega}$ at $q$ is formally linearizable but non-resonant, it follows that $\hat{\Omega}$ extends as a transversely formal closed 1 -form to $D_{q}$. This shows that $\hat{\eta}$ extends to $q$.

(3) $\tilde{\omega}$ at $q$ is resonant but not formally linearizable. Here we notice that the local holonomy associated to the separatrix $S$ at $q$ is (tangent to 1 ) of the form

$$
\varphi(z)=z /\left(1+a z^{k}\right)^{1 / k}
$$

for some formal coordinate $z$. In fact, we know that any homography which is not tangent to 1 is linearizable, and on the other hand, the linearization on the local holonomy implies the linearization of the form $\tilde{\omega}$ at the singularity ([22]). Therefore, this holonomy coincides with the holonomy of the germ of $\omega_{k, l}$ at singularity

$$
\omega_{k, l}=l x d y+k y\left[1+(\sqrt{-1} / 2 \pi) x^{k} y^{l}\right] d x=0 .
$$

Thus (see [23], [9], [33]) the foliation $\tilde{\omega}=0$ at the singularity $q$ is formally conjugate to the foliation $\omega_{k, l}=0$. Therefore there are formal coordinates $(\hat{x}, \hat{y})$ centered at $q$ such that for some formal meromorphic function $\hat{g}$ we have $\tilde{\omega}(\hat{x}, \hat{y})=\hat{g} \omega_{k, l}(\hat{x}, \hat{y})$. Moreover, if we define

$$
\hat{\eta}_{o}=(k+1) d \hat{y} / \hat{y}+(l+1) d \hat{x} / \hat{x}+d \hat{g} / \hat{g},
$$

then we obtain $d \tilde{\omega}=\hat{\eta}_{o} \wedge \tilde{\omega}$. We have $\hat{\eta}-\hat{\eta}_{o}=\hat{h} \tilde{\omega}$ for some formal expression $\hat{h}$, which satisfies $d(\hat{h} \tilde{\omega})=0$. On the other hand, we know that by construction, $\operatorname{Res}_{\Lambda_{o}} \eta=k+1$, so that $\hat{h} \tilde{\omega}$ is closed, and holomorphic along $\Lambda_{o} \backslash\{q\}$. Since $\tilde{\omega}$ at the singularity $q$ is of the (nonlinearizable) formal normal form above, it follows that $\hat{h} \tilde{\omega}=0$. Therefore we extend $\hat{\eta}$ as $\hat{\eta}_{o}$ to $q$.

Thus we have constructed a transversely formal $\hat{\eta}$ over the curve $\Lambda$. In the same way, we may construct $\hat{\eta}$ over the divisor $D$ ([8], [33]). Now, Hironaka-Matsumura theorem ([17]) asserts that both $\hat{h}$ and $\hat{\eta}$, constructed in the lemma above, extend meromorphically to $\boldsymbol{C} P(2)$ ([11], [9]). This proves Lemma 8.

Finally, we use the following

LEMMA 9. If all singularities are resonant, then $\eta$ has entire residues. If there is some nonresonant singularity, then each virtual holonomy group is abelian.

PROOF. If all singularities are resonant, then using the construction given for $\hat{\eta}$ above, we conclude that it has entire residues. Now we use the following

Claim 3. Assume that there exists some nonresonant singularity $q \in D$. Then each virtual holonomy group associated to a component of $D$ contains some diffeomorphism whose linear part is nonperiodic. In particular, all virtual holonomy groups are abelian. 
Indeed, let $q \in D_{j_{o}}$ and $D_{j}$ be such that $q_{j}=D_{j} \cap D_{j_{o}} \neq \emptyset$. Then we have two possibilities for $q_{j}$. If $q_{j}$ is nonresonant, then its local holonomy associated to $D_{j}$ induces the desired diffeomorphism for $D_{j}$. If $q_{j}$ is resonant, then we may use the adjunction process to "pass" the nonresonant diffeomorphism given by $q$ from the virtual holonomy of $D_{j_{o}}$ to the virtual holonomy of $D_{j}$. This induced element is still nonresonant by the construction of the adjunction given in Section 2. Thus we may reach all the components that are adjacent to $D_{j_{o}} \cup D_{j}$ and so on. Using the fact that the invariant part of $D$ is connected, we conclude that all virtual holonomy groups contain elements with nonperiodic linear part. According to [20], a subgroup of $\operatorname{Diff}(\boldsymbol{C}, 0)$ with discrete pseudo-orbits off the origin and containing such nonresonant elements must be abelian.

Using Lemma 9 and the first part of the Lemma 8, we may assume that all singularities $q \in \Lambda$ are resonant so that $\hat{\eta}$ has simple poles and entire residues, all of which are along $\Lambda$ and the separatrices through these points $q$ are transverse to $\Lambda$. According to the integration lemma ([31]), this implies that $\eta=d H / H$ for some rational function $H$ and therefore $\tilde{\mathcal{F}}(X)$ is given by the closed rational 1 -form $\tilde{\Omega}=\tilde{\omega} / H$. The proof of Proposition 8 is now complete.

6. Compact foliations with singularities. One of the basic questions concerning compact foliations in the classical framework is: If a compact manifold is foliated by compact submanifolds in a smooth way, is there any upper bound on the volume of the leaves? The positive answer to this question was given by Sullivan and Edwards-Millett-Sullivan ([12]) under some mild orientability hypothesis on the foliation for the real (nonsingular) case. In this last section we consider several related problems motivated by our results and some classical results of the theory of foliations.

PROPOSITION 9. Let $\mathcal{F}$ be a $C^{2}$ codimension one (real nonsingular) foliation on a compact connected manifold $M$ of dimension three with $\pi_{1}(M)$ finite. Assume that for some $C^{2}$ metric on $M$ the leaves are nonpositively curved minimal surfaces with finite total curvature. Then the following hold:

(1) There exists a compact leaf.

(2) The union $\Sigma$ of the flat leaves is compact and invariant.

(3) The leaves are closed outside $\Sigma$.

(4) If $\Sigma$ is empty, then all leaves are compact and with finite holonomy groups.

(5) If there exists some nonflat compact leaf $L_{o}$, then all leaves are compact with finite holonomy groups.

Proof. (1) follows from Novikov theorem ([4]). According to Lemma 7, the leaves having finite total curvature are closed outside flat leaves. Therefore, $\Sigma$ is closed, and hence compact, proving (2) and (3). If $\Sigma=\emptyset$, then by (3) the leaves are closed and hence compact. Using [12], we conclude that all the holonomy groups are finite, proving (4).

Let now $L_{o}$ be a compact nonflat leaf. Then since $\Sigma$ is closed, it follows that there exists a certain relatively compact open neighborhood $V$ of $L_{o}$ such that $V \cap \Sigma=\emptyset$. Since $\Sigma$ is 
invariant, we can take $V$ to be invariant and choose an invariant subneighborhood $L_{o} \subset W \subset$ $\bar{W} \subset V$. In particular, for any $x \in W$ the leaf $L_{x} \ni x$ is closed and contained in $\bar{W}$. Thus $L_{x}$ is compact for all $x \in W$. Therefore, since $\mathcal{F}$ has codimension one, it follows from a result of Reeb ([30]) that all the volume of the leaves meeting $\bar{W}$ is uniformly bounded and therefore the holonomy groups of these leaves are finite. In particular, $L_{o}$ has a finite holonomy group. Using now Reeb stability theorem ([4]) for codimension one foliations, we conclude that all the leaves are compact and with finite holonomy groups.

If we take a complex Kaehlerian surface $M$ equipped with a singular holomorphic foliation $\mathcal{F}$, then all leaves of $\mathcal{F}$ are minimal immersed submanifolds and therefore nonpositively curved. Examples are given by rational 1-forms defining algebraic foliations on complex projective surfaces. On the other hand, the additional restriction of finite curvature is quite restrictive as seen by our next result.

PROPOSITION 10. Let $\mathcal{F}$ be a dimension one singular holomorphic foliation on a complex manifold $M$ of dimension two, equipped with a Hermitian metric $g$ which makes the leaves nonpositively and finitely curved. Denote by $\Sigma \subset M \backslash \operatorname{sing} \mathcal{F}$ the union of flat leaves, and assume that $\mathcal{F}$ has some nonflat leaf. Then we have two possibilities:

(i) The holonomy groups of the leaves are solvable with discrete pseudo-orbits outside the origin.

(ii) $\Sigma$ is not transversely discrete. If moreover the metric $g$ is real analytic, then all the leaves are flat.

Proof. Assume that $\Sigma$ is transversely discrete. Let $L_{o}$ be a leaf of $\mathcal{F}$. If $L_{o} \not \subset \Sigma$, then $L_{o} \cap \Sigma=\emptyset$, and given any sufficiently small transverse disc $D \cap L_{o}=\{q\} \notin \operatorname{sing} \mathcal{F}$ we have $D \cap \Sigma=\emptyset$. This implies, by Lemma 7, that the holonomy group $\operatorname{Hol}\left(\mathcal{F}, L_{o}, D\right)$ has finite orbits and therefore it is a finite group as we have already seen. If $L_{o} \subset \Sigma$, then, since $\Sigma$ is transversely discrete, given a small transverse disc $D$ as above we have $\Sigma \cap D=\Sigma \cap L_{o}$, which is a point. Therefore, again $\operatorname{Hol}\left(\mathcal{F}, L_{o}, D\right)$ has finite orbits and is therefore finite.

Assume now that $\Sigma$ is not transversely discrete, and let $L_{o}$ be some leaf with nonsolvable holonomy group. If $L_{o}$ is accumulated by $\Sigma$, then using Nakai density theorem ([26]), we conclude that $\Sigma$ is locally dense around $L_{o}$. In this case, if we also assume that the metric $g$ is real analytic, then $\Sigma$ is transversely real analytic and therefore $\Sigma=M \backslash \operatorname{sing} \mathcal{F}$. If $L_{o}$ is not accumulated by $\Sigma$, then we may choose as above a small disc $D$ transverse to $\mathcal{F}$ with $D \cap \Sigma \subset D \cap L_{o}$. This implies that $\operatorname{Hol}\left(\mathcal{F}, L_{o}, D\right)$ has finite orbits and is therefore finite.

The following proposition is proved in a similar way to Proposition 10.

PROPOSITION 11. Let $\mathcal{F}$ be a real analytic codimension one singular foliation on a connected (real) manifold $M$ of dimension three, such that there exists an analytic Riemannian metric for which the leaves are nonpositively curved and with finite total curvature. If $\mathcal{F}$ has some nonflat leaf, then all leaves have solvable holonomy groups (with discrete pseudo-orbits outside the origin). Moreover, if $M$ is compact, $\mathcal{F}$ is nonsingular and without flat leaves, then all leaves are compact with finite holonomy groups. 
Proof. Let $L_{o}$ be a leaf of $\mathcal{F}$. If $L_{o}$ is not accumulated by $\Sigma$, then we may choose a small transverse section $I \approx(-1,1)$ with $I \cap \Sigma=L_{o} \cap \Sigma$. Denote by $\operatorname{Diff}^{w}(\boldsymbol{R}, 0)$ the group of germs of one dimensional real analytic diffeomorphisms fixing the origin. Therefore, the holonomy group $\operatorname{Hol}\left(\mathcal{F}, L_{o}, I\right) \subset \operatorname{Diff}^{w}(\boldsymbol{R}, 0)$ has some orbits which are discrete and it must be a solvable group ([26]). If $L_{o}$ is accumulated by $\Sigma$ and $\operatorname{Hol}\left(\mathcal{F}, L_{o}, I\right)$ is not solvable for some small transverse section $I$, then $\Sigma \cap I$ is dense in $I$ and therefore $\Sigma \cap I=I$. Since the metric is real analytic, $\Sigma$ is transversely real analytic and $\Sigma \cap I=I$ implies that $\Sigma=M \backslash \operatorname{sing} \mathcal{F}$, a contradiction.

Assume now that $M$ is compact, $\mathcal{F}$ is nonsingular and $\Sigma$ is empty. Then any leaf $L_{o}$ of $\mathcal{F}$ is compact, because it is closed by Lemma 7. Therefore $\mathcal{F}$ is nonsingular real analytic and all its leaves are compact. This implies that the holonomy groups are finite, since they have finite orbits.

The following result gives a characterization of compact complex foliations with singularities in terms of the total curvature.

PROPOSITION 12. Let $\mathcal{F}$ be a holomorphic (singular) foliation by curves on a compact complex manifold $M$ of dimension two equipped with a $C^{2}$ Riemannian metric $g$ of nonpositive curvature. Then the following conditions are equivalent:

(i) There exist $\delta>0$ and $C>0$ with $-\delta>\int_{L} K d \sigma>-C$ for all leaves $L$.

(ii) Every leaf is nonflat, with compact analytic closure of complex dimension one, and finite holonomy group.

Proof. We only prove that (ii) implies (i). Let $L_{o}$ be a leaf of $\mathcal{F}$. There are no flat leaves so that $L_{o}$ is closed outside the singular set $\operatorname{sing} \mathcal{F}$ (Lemma 7). According to RemmertStein theorem ([16]), the closure $\overline{L_{o}}$ is an analytic curve and, since $M$ is compact, $\overline{L_{o}}$ is a compact curve. Therefore all leaves have compact closures. Given any leaf $L$ and a small transverse disc $\boldsymbol{D}$ with $\boldsymbol{D} \cap L=p$, the holonomy group $\operatorname{Hol}(\mathcal{F}, L, \boldsymbol{D}, p)$ is a subgroup of $\operatorname{Diff}(\boldsymbol{D}, p) \cong \operatorname{Diff}(\boldsymbol{C}, 0)$ with finite pseudo-orbits (for the leaves are compact), and this implies by [22] that this group is finite. The converse is left to the reader.

\section{REFERENCES}

[ 1 ] J. C. Alexander And A. Verjovsky, First integrals for singular holomorphic foliations with leaves of bounded volume, Holomorphic dynamics (Mexico, 1986), 1-10, Lecture Notes in Math. 1345, SpringerVerlag, Berlin-New York, 1988.

[2] M. Brunella, Foliations on the complex projective plane with many parabolic leaves, Ann. Inst. Fourier (Grenoble) 44 (1994), 1237-1242.

[ 3 ] C. CAмасно, On the local structure of conformal maps and holomorphic vector fields in $\boldsymbol{C}^{2}$, Jounées Singuliéres de Dijon (Univ. Dijon, Dijon, 1978), 3, 83-94, Astérisque 59-60, Soc. Math. France, Paris, 1978.

[4] C. Camacho and A. Lins Neto, Geometric theory of foliations, Birkhauser Inc., Boston, Mass., 1985.

[ 5 ] C. CAmacho, A. Lins Neto And P. SAD, Foliations with algebraic limit sets, Ann. of Math. (2) 136 (1992), 429-446.

[6] C. CAmacho, A. Lins Neto and P. SAD, Minimal sets of foliations on complex projective spaces, Publ. Math. Inst. Hautes Études Sci. 68 (1988), 187-203.

[ 7 ] C. CAMACHO AND P. SAD, Invariant varieties through singularities of holomorphic vector fields, Ann. of Math. 115 (1982), 579-595. 
[ 8 ] C. CAMACho AND B. Azevedo SCÁRdua, Holomorphic foliations with Liouvillian first integrals, Ergodic Theory Dynam. Systems 21 (2001), 717-756.

[ 9 ] C. CAmacho And B. Azevedo SCÁRduA, Foliations on complex projective spaces with algebraic limit sets. Géométrie complexe et systèmes dynamiques (Orsay, 1995), Astérisque, 261, 57-88, Soc. Math. France, Paris, 2000

[10] F. CANO AND D. CERVEAU, Desingularization of holomorphic foliations and existence of separatrices, Acta Math. 169 (1992), 1-103.

[11] D. Cerveau and B. Azevedo ScÁrdua, Complete polynomial vector fields in the affine space of two complex variables, Pre-print, IMPA-Univ. Rennes 1, 1999.

[12] R. EdWARds, K. Millet AND D. Sullivan, Foliations with all leaves compact, Topology 16 (1977), $13-32$.

[13] D. B. A. Epstein, Foliations with all leaves compact, Ann. Inst. Fourier, Grenoble 26 (1976), 265-282.

[14] H. Grauert and R. Remmert, Theory of Stein Spaces, Springer-Verlag, Berlin-New York, 1979.

[15] R. C. GunNing, Introduction to holomorphic functions of several variables, vol. I, Function Theory. Wadsworth \& Brooks/Cole Advanced Books \& Software, Pacific Grove, CA, 1990.

[16] R. C. GUNNING, Introduction to holomorphic functions of several variables, vol. II, Local Theory. Wadsworth \& Brooks/Cole Advanced Books \& Software, Monterey, CA, 1990.

[17] H. HironaKa AND H. Matsumara, Formal functions and formal embeddings, J. Math. Soc. Japan 20 (1968), 52-82.

[18] A. Huber, On subharmonic functions and differential geometry in the large, Comment. Math. Helv. 32 (1957), 13-72.

[19] J. P. JouAnolou, Équations de Pfaff algèbriques, Lecture Notes in Math. 708, Springer-Verlag, Berlin, 1979.

[20] A. Lins Neto And B. Azevedo ScÁrdua, Folheaçoes Algébricas Complexas, book of the XXI Colóquio Brasileiro de Matemática-IMPA, 1997.

[21] F. LORAY, Feuilletages holomorphes à holonomie résoluble, These Université de Rennes I, 1994.

[22] J. F. Mattei And R. Moussu, Holonomie et intégrales premières, Ann. Sci. École Norm. Sup. (4) 13 (1980), 469-523.

[23] J. MARTINET AND J-P. RAMIS, Classification analytique des équations différentielles non linéaires resonnants du premier ordre, Ann. Sci. École Norm. Sup. (4) 16 (1983), 571-621.

[24] J. Martinet And J-P. Ramis, Problème de modules pour des équations différentielles non linéaires du premier ordre, Publ. Math. Inst. Hautes Études Scientifiques 55 (1982), 63-124.

[25] R. S. MoL, Meromorphic first integrals: some extension results, Tohoku Math. J. 54 (2002), 85-104.

[26] I. NAKAI, Separatrices for nonsolvable dynamics on C, 0, Ann. Inst. Fourier (Grenoble) 44 (1994), 569-599.

[27] R. OsSERMAN, Global properties of minimal surfaces in $E^{3}$ and $E^{n}$, Ann. of Math. (2) 80 (1964), 340-364.

[28] J. F. Plante, A generalization of the Poincaré-Bendixon Theorem for foliations of codimension one, Topology 12 (1973), 177-181.

[29] J. F. Plante, Foliations with measure preserving holonomy, Ann. of Math. (1975), 327-361.

[30] G. ReEB, Sur certaines propriétés topologiques des varietés feuilletées, Publ. Inst. Math. Univ. Strasbourg 11, 5-89, 155-156, Actualités Sci. Ind. 1183, Hermann, Paris, 1952.

[31] B. AzEvedo SCÁRduA, Transversely affine and transversely projective foliations, Ann. Sc. École Norm Sup. (4) 30 (1997), 169-204.

[32] B. AzEvedo SCÁRduA, Polynomial foliations with sub-exponential growth, Indag. Math. (N.S.) 12 (2001), 293-302.

[33] B. AzEvedo SCÁRDUA, Integration of complex differential equations, J. Dynam. Control Systems 5 (1999), $1-50$.

[34] B. Azevedo ScárduA, A Remark on Parabolic Projective Foliations, Hokkaido Math. J. 28 (1999), 231252.

[35] A. Seidenberg, Reduction of singularities of the differential equation $A d y=B d x$, Amer. J. Math. 90 (1968), 248-269.

[36] Y. SiU, Techniques of Extension of Analytic Object, Lecture Notes in Pure and Appl. Math. 8, Marcel Dekker, Inc., New York, 1974 
[37] M. Suzuki, Sur les opérations holomorphes de $\boldsymbol{C}$ et de $\boldsymbol{C}^{*}$ sur un space de Stein, Fonctions de pluiseurs variables complexes, III, (Sém. François Norguet, 1975-1977), 80-88, Lecture Notes in Math. 670, Berlin, 1978.

[38] B. White, Complete surfaces of finite total curvature, J. Differential Geom. 26 (1987), 315-326.

[39] B. WiRTZ, Entropy and fixed points for nonsolvable dynamics of $(\boldsymbol{C}, 0)$, Bol. Soc. Brasil. Mat. (N.S.) 29 (1998), 53-74.

INSTITUTO DE MATEMÁTICA

UNIVERSIDADE FEDERAL DO RIO DE JANEIRO

CaIXa Postal 68530

21945-970 RIO DE JANEIRO-RJ

BRAZIL

E-mail address: scardua@im.ufrj.br 